\title{
INPUT-TO-STATE STABILITY OF DIFFERENTIAL INCLUSIONS WITH APPLICATIONS TO HYSTERETIC AND QUANTIZED FEEDBACK SYSTEMS*
}

\author{
BAYU JAYAWARDHANA ${ }^{\dagger}$, HARTMUT LOGEMANN $^{\dagger}$, AND EUGENE P. RYAN ${ }^{\dagger}$
}

SICON 071132: December 2007, revised June 2008

\begin{abstract}
Input-to-state stability (ISS) of a class of differential inclusions is proved. Every system in the class is of Lur'e-type: a feedback interconnection of a linear system and a set-valued nonlinearity. Applications of the ISS results, in the context of feedback interconnections with a hysteresis operator or a quantization operator in the feedback path, are developed.
\end{abstract}

Key words. Absolute stability, circle criterion, differential inclusions, input-to-state stability, hysteresis, nonlinear systems, quantization.

AMS subject classifications. 34A60, 34C55, 34D05, 47J40, 93C10, 93D05, 93D10.

1. Introduction. Classical absolute stability theory, with origins in [18], is concerned with the analysis of systems of Lur'e type, that is, feedback interconnections, of the form shown in Figure 1.1, consisting of a linear system $L$ in the forward path and a static sector-bounded nonlinearity $f$ in the (negative) feedback path. The methodology seeks to conclude stability of the overall system through the interplay or reciprocation of inherent frequency-domain properties of the linear component $L$ and sector data for the nonlinearity $f$. Accounts of the classical theory can be found in, e.g., [7, 10, 13, 19, 21, 23].

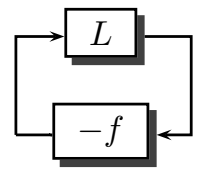

FIG. 1.1. Classical feedback interconnection

The present paper adopts a similar standpoint, but differs from the classical framework in three fundamental aspects: (i) in contrast with the literature, wherein the focus is on global asymptotic stability and $L^{2}$ or $L^{\infty}$ stability, input-to-state stability issues are addressed here; (ii) nonlinearities of considerably greater generality are permitted in the feedback path; (iii) the sector conditions of the classical theory are significantly weakened. With reference to (i), conditions on the linear and nonlinear components are identified under which input-to-state stability of the interconnection is guaranteed. With reference to (ii), a framework is developed of sufficient generality to encompass not only static nonlinearities but also causal operators (and hysteresis, in particular) and quantization operators in the feedback path. With reference to (iii), through the concept of a generalized sector condition, the investigation is extended to include nonlinearities which satisfy a sector condition only in the complement of a compact set: a theory is developed pertaining to input-to-state stability with bias of the feedback interconnection. We proceed to outline these features more precisely.

With reference to Figure 1.2, the focus of the paper is a study of absolute stability, input-to-state stability, and boundedness properties of a feedback interconnection of a finite-dimensional, linear, $m$-input, $m$-output system $(A, B, C)$ and

\footnotetext{
* Supported by the UK Engineering \& Physical Sciences Research Council (Grant Ref: GR/S94582/01).

${ }^{\dagger}$ Department of Mathematical Sciences, University of Bath, Bath BA2 7AY, UK. B Jayawardhana is now with the Department of Discrete Technology and Production Automation, University of Groningen, 9747 AG Groningen, The Netherlands.

Email: B.Jayawardhana@rug.nl, hl@maths.bath.ac.uk, epr@maths.bath.ac.uk
} 
a set-valued nonlinearity $\Phi$. Throughout, we assume that $\Delta$ is a set-valued map in which input or disturbance signals are embedded. We seek an analytical framework

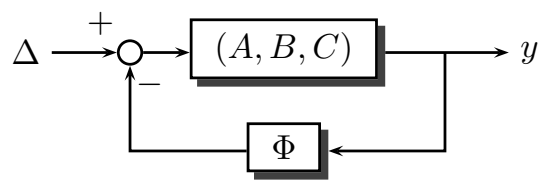

FIG. 1.2. Interconnection of a linear system $(A, B, C)$ and a set-valued nonlinearity $\Phi$

of sufficient generality to encompass inter alia feedback systems with causal operators (and, in particular, hysteresis operators) in the feedback loop. To illustrate this, let $F$ be a causal operator from $\operatorname{dom}(F) \subset L_{\text {loc }}^{1}\left(\mathbb{R}_{+}, \mathbb{R}^{m}\right)$ to $L_{\text {loc }}^{1}\left(\mathbb{R}_{+}, \mathbb{R}^{m}\right)$, where $\mathbb{R}_{+}:=[0, \infty)$, and consider the feedback system (structurally of Lur'e type), with input $d \in L_{\text {loc }}^{\infty}\left(\mathbb{R}_{+}, \mathbb{R}^{m}\right)$, given by the functional differential equation

$$
\dot{x}(t)=A x(t)+B(d(t)-(F(C x))(t)) .
$$

By causality of $F$ we mean that, for all $y, z \in \operatorname{dom}(F)$ and all $\alpha>0$,

$$
\left.y\right|_{[0, \alpha]}=\left.\left.z\right|_{[0, \alpha]} \Longrightarrow F(y)\right|_{[0, \alpha]}=\left.F(z)\right|_{[0, \alpha]} \text {. }
$$

To associate (1.1) with the structure of Figure 1.2, assume that $F$ can be embedded in a set-valued map $\Phi$ in the sense that

$$
y \in \operatorname{dom}(F) \quad \Longrightarrow \quad(F(y))(t) \in \Phi(y(t)) \text { for a.e. } t \in \mathbb{R}_{+} .
$$

If the input $d$ is such that $d(t) \in \Delta(t)$ for almost all $t$, then any solution of (1.1) is a fortiori a solution of the feedback interconnection in Figure 1.2. In this sense, properties of solutions of the feedback interconnection are inherited by solutions of (1.1). Under particular regularity assumptions on $\Delta$ and $\Phi$, generalized sector conditions on $\Phi$, and positive-real conditions related to the linear component $(A, B, C)$, we establish input-to-state stability (in the sense of [20], but extended to differential inclusions) and boundedness properties of solutions of the system in Figure 1.2. The approach is partially based on that of Arcak \& Teel [1]: in particular, some of the arguments adopted in the proof of Lemma 5.1 of the present paper are generalizations, to a differential inclusions setting, of arguments in [1]. The paper is structured as follows. In Section 2, we make precise the nature of the maps $\Phi$ and $\Delta$ and state an existence theorem which underpins the stability analysis of the differential inclusion formulation implicit in Figure 1.2. The main results, Theorems 3.4 and 3.5 (and Corollaries 3.6 and 3.7), are assembled in Section 3. For clarity of presentation, the proof of Theorem 3.4 (respectively, Theorem 3.5 ) is presented separately in Section 4 (respectively, Section 5). In Section 6, the results in Theorem 3.4/Corollary 3.6 are applied in the context of single-input, single-output feedback interconnections with a hysteresis operator $F$ in the feedback loop. New absolute stability and boundedness results are obtained for Lur'e systems with Preisach hysteresis (see e.g. $[3,9,12,16,17]$ for earlier stability results for hysteretic feedback systems). In the final section, quantized feedback systems are considered: these constitute an area of growing importance (see e.g. $[4,8]$ in a linear systems context). Specifically, in Section 7, nonlinear feedback systems with uniform output quantization (parameterized by $\gamma \geq 0$ ) are investigated. Through an application of Theorem 3.5/Corollary 3.7, we establish robustness with respect to quantization in the following sense: if, in the absence of quantization $(\gamma=0)$, the feedback system is input-to-state stable (ISS), then, in the presence of quantization $(\gamma>0)$, the feedback system is ISS with bias and is such that the unbiased ISS property of the unquantized system is "approached" as $\gamma \downarrow 0$. 
Notation and terminology. The open right-half complex plane is denoted by $\mathbb{C}_{+}$. For non-empty $S \subset \mathbb{R}^{m}$, we define $|S|:=\sup \{\|s\| \mid s \in S\}$. If $H$ is a proper real-rational matrix of format $m \times m$, then we say that $H$ is positive real if

$$
H(s)+H^{*}(s) \geq 0, \quad \forall s \in \mathbb{C}_{+}, s \text { not a pole of } H,
$$

where $H^{*}(s):=(H(s))^{*}$. Moreover, if $H \in H^{\infty} \equiv H^{\infty}\left(\mathbb{C}_{+}, \mathbb{C}^{m \times m}\right)$ (and so $H$ does not have any poles in $\left.\overline{\mathbb{C}}_{+}\right)$, then

$$
\|H\|_{H^{\infty}}:=\sup _{s \in \mathbb{C}_{+}}\|H(s)\|,
$$

where $\|H(s)\|$ is the matrix norm induced by the 2 -norm on $\mathbb{C}^{m}$. Let $\mathcal{K}$ denote the set of all continuous and strictly increasing functions $f: \mathbb{R}_{+} \rightarrow \mathbb{R}_{+}$with $f(0)=0$. We say that a function $f$ is in $\mathcal{K}_{\infty}$ if $f \in \mathcal{K}$ and $f(s) \rightarrow \infty$ as $s \rightarrow \infty$. Finally, $\mathcal{K} \mathcal{L}$ denotes the class of all continuous functions $f: \mathbb{R}_{+}^{2} \rightarrow \mathbb{R}_{+}$such that, for each $r \in \mathbb{R}_{+}$, the function $s \mapsto f(r, s)$ is in $\mathcal{K}$ and, for each $s \in \mathbb{R}_{+}$, the function $r \mapsto f(r, s)$ is non-increasing with $f(r, s) \rightarrow 0$ as $r \rightarrow \infty$.

2. Set-valued nonlinearities and differential inclusions. A set-valued map $y \mapsto \Phi(y) \subset \mathbb{R}^{m}$, with non-empty values and defined on $\mathbb{R}^{m}$, is said to be upper semicontinuous at $y \in \mathbb{R}^{m}$ if, for every open set $U$ containing $\Phi(y)$, there exists an open neighbourhood $Y$ of $y$ such that $\Phi(Y):=\cup_{z \in Y} \Phi(z) \subset U$; the map $\Phi$ is said to be upper semicontinuous if it is upper semicontinuous at every $y \in \mathbb{R}^{m}$. The set of upper semicontinuous compact-convex-valued maps

$$
\Phi: \mathbb{R}^{m} \rightarrow\left\{S \subset \mathbb{R}^{m} \mid S \text { non-empty, compact and convex }\right\}
$$

is denoted by $\mathcal{U}$. Let $\Delta: \mathbb{R}_{+} \rightarrow\left\{S \subset \mathbb{R}^{m} \mid S \neq \emptyset\right\}$ be a set-valued map. The map $\Delta$ is said to be measurable if the preimage $\Delta^{-1}(U):=\left\{t \in \mathbb{R}_{+} \mid \Delta(t) \cap U \neq \emptyset\right\}$ of every open set $U \subset \mathbb{R}^{m}$ is Lebesgue measurable; $\Delta$ is said to be locally essentially bounded if $\Delta$ is measurable and the function $t \mapsto|\Delta(t)|$ is in $L_{\text {loc }}^{\infty}\left(\mathbb{R}_{+}\right)$. The set of all locally essentially bounded set-valued maps $\mathbb{R}_{+} \rightarrow\left\{S \subset \mathbb{R}^{m} \mid S \neq \emptyset\right\}$ is denoted by $\mathcal{B}$. For $\Delta \in \mathcal{B}, I \subset \mathbb{R}_{+}$an interval and $1 \leq p \leq \infty$, the $L^{p}$-norm of the restriction of the function $t \mapsto|\Delta(t)|$ to the interval $I$ is denoted by $\|\Delta\|_{L^{p}(I)}$. For later use, we record a technicality.

Lemma 2.1. Assume that $\Phi \in \mathcal{U}, \Phi(0)=\{0\}$ and there exists $\varphi \in \mathcal{K}_{\infty}$ with

$$
\varphi(\|y\|)\|y\| \leq\langle y, v\rangle \quad \forall v \in \Phi(y), \forall y \in \mathbb{R}^{m} .
$$

Then there exists $\psi \in \mathcal{K}_{\infty}$ such that

$$
\|v\| \leq \psi(\|y\|) \quad \forall v \in \Phi(y), \forall y \in \mathbb{R}^{m} .
$$

Proof. By upper semicontinuity of $\Phi$ and compactness of its values, for every compact set $K \subset \mathbb{R}^{m}$, the set $\Phi(K)$ is compact (see, for example, [2, Proposition 3 , p. 42]), and so the function $s \mapsto \psi_{0}(s):=\max \{\|v\| \mid v \in \Phi(y),\|y\| \leq s\}$ is well defined and non-decreasing on $\mathbb{R}_{+}$, with $\psi_{0}(0)=0$. Clearly, $\varphi(s) \leq \psi_{0}(s)$ for all $s \in \mathbb{R}_{+}$and so $\psi_{0}(s) \rightarrow \infty$ as $s \rightarrow \infty$. Let $\psi \in \mathcal{K}_{\infty}$ be such that $\psi(s) \geq \psi_{0}(s)$ for all $s \in \mathbb{R}_{+}$, for example, the function $\psi \in \mathcal{K}_{\infty}$ given by

$$
\psi(0):=0, \quad \psi(s):=\frac{1}{s} \int_{s}^{2 s} \psi_{0}(\sigma) d \sigma, \quad \forall s>0
$$

suffices.

The feedback system shown in Figure 1.2 corresponds to the initial-value problem

$$
\dot{x}(t)-A x(t) \in B(\Delta(t)-\Phi(C x(t))), \quad x(0)=x^{0} \in \mathbb{R}^{n}, \quad \Delta \in \mathcal{B},
$$


where $A \in \mathbb{R}^{n \times n}, B \in \mathbb{R}^{n \times m}, C \in \mathbb{R}^{m \times n}$ and $\Phi \in \mathcal{U}$. By a solution of (2.1) we mean an absolutely continuous function $x:[0, \omega) \rightarrow \mathbb{R}^{n}, 0<\omega \leq \infty$, such that $x(0)=x^{0}$ and the differential inclusion in (2.1) is satisfied almost everywhere on $[0, \omega)$; a solution is maximal if it has no proper right extension that is also a solution; a solution is global if it exists on $[0, \infty)$. Before developing a stability theory for systems of the form (2.1), we briefly digress to record an existence result.

LEMmA 2.2. Let $\Phi \in \mathcal{U}$. For each $x^{0} \in \mathbb{R}^{n}$ and each $\Delta \in \mathcal{B}$, the initial-value problem (2.1) has a solution. Moreover, every solution can be extended to a maximal solution $x:[0, \omega) \rightarrow \mathbb{R}^{n}$ and, if $x$ is bounded, then $x$ is global.

Proof. Let $x^{0} \in \mathbb{R}^{n}$ and $\Delta \in \mathcal{B}$ be arbitrary. By [6, Corollary 5.2], the initialvalue problem (2.1) has a solution and every solution can be extended to a solution $x:[0, \omega) \rightarrow \mathbb{R}^{n}$ with the property that the graph of $x$ is unbounded. Evidently, $x$ is maximal and, if $x$ is bounded, then $\omega=\infty$.

3. Input-to-state stability: the main results. In the context of the differential inclusion (2.1), the transfer-function matrix of the linear system given by $(A, B, C)$ is denoted by $G$, i.e., $G(s)=C(s I-A)^{-1} B$.

We assemble four hypotheses which will be variously invoked in the theory developed below.

(H1) There exist numbers $a<b$ and $\delta>0$ such that

$$
\langle a y-v, b y-v\rangle \leq 0 \quad \forall v \in \Phi(y), \forall y \in \mathbb{R}^{m},
$$

$G(I+a G)^{-1} \in H^{\infty}$ and $(I+b G)(I+a G)^{-1}-\delta I$ is positive real.

(H2) $\Phi(0)=\{0\}$ and there exist numbers $a>0, \delta \in[0,1)$ and $\theta \geq 0$ such that

$$
\begin{aligned}
a\|y\|^{2} & \leq\langle y, v\rangle \quad \forall v \in \Phi(y), \forall y \in \mathbb{R}^{m}, \\
\|v-a \delta y\| & \leq\langle y, v-a \delta y\rangle \quad \forall v \in \Phi(y), \forall y \in \mathbb{R}^{m} \text { with }\|y\| \geq \theta
\end{aligned}
$$

and $G(I+\delta a G)^{-1}$ is positive real.

(H3) There exist $\varphi \in \mathcal{K}_{\infty}$ and numbers $b>0$ and $\delta \in[0,1)$ such that

$$
\max \left\{\varphi(\|y\|)\|y\|,\|v\|^{2} / b\right\} \leq\langle y, v\rangle \quad \forall v \in \Phi(y), \forall y \in \mathbb{R}^{m}
$$

and $(\delta / b) I+G$ is positive real.

(H4) $\Phi(0)=\{0\}$ and there exist $\varphi \in \mathcal{K}_{\infty}$ and a number $\theta \geq 0$ such that

$$
\begin{aligned}
\varphi(\|y\|)\|y\| \leq\langle y, v\rangle & \forall v \in \Phi(y), \forall y \in \mathbb{R}^{m}, \\
\|v\| \leq\langle y, v\rangle & \forall v \in \Phi(y), \forall y \in \mathbb{R}^{m} \text { with }\|y\| \geq \theta
\end{aligned}
$$

and $G$ is positive real.

REMARK 3.1. (a) (H1) is a set-valued version of the familiar multivariable sector condition. A routine calculation shows that (3.1) holds if and only if

$$
\left\|v-\frac{a+b}{2} y\right\| \leq \frac{b-a}{2}\|y\| \quad \forall v \in \Phi(y), \forall y \in \mathbb{R}^{m} .
$$

(b) If $m=1$ (the single-input, single-output case), then the combined frequencydomain assumptions in (H1), namely the condition $G(I+a G)^{-1} \in H^{\infty}$ together with the positive realness of $(I+b G)(I+a G)^{-1}-\delta I$, admit a graphical characterization in terms of the Nyquist diagram of $G$ (see, e.g., [13, pp. 268]).

(c) Conditions (3.2) and (3.5) can be viewed as the limits of (3.1) and (3.4), respectively, as $b \rightarrow \infty$.

(d) A sufficient condition for (3.4) to hold is the "nonlinear" sector condition

$$
\left\langle\varphi(y)\|y\|^{-1} y-v, b y-v\right\rangle \leq 0 \quad \forall v \in \Phi(y), \forall y \in \mathbb{R}^{m},
$$


which is (3.1) with the term ay replaced by $\varphi(y)\|y\|^{-1} y$ (which should be interpreted as taking the value 0 for $y=0$ ). It is easy to construct counterexamples which show that (3.7) is not necessary for (3.4) to hold.

(e) If $m=1$ and (3.2) holds, then (3.3) is trivially satisfied for any $\theta \geq 1$ and any $\delta \in[0,1)$. Similarly, if $m=1$ and (3.5) holds, then (3.6) is satisfied for every $\theta \geq 1$.

(f) If (3.4) holds for some $\varphi \in \mathcal{K}_{\infty}$ and for some $b>0$, then $\Phi(0)=\{0\}$ and, furthermore, (3.6) is satisfied for any $\theta>0$ satisfying $\varphi(\theta) \geq b$.

Definition 3.2. System (2.1) is said to be input-to-state stable with bias $c \geq 0$ if every maximal solution of (2.1) is global, and there exist $\beta_{1} \in \mathcal{K} \mathcal{L}$ and $\beta_{2} \in \mathcal{K}_{\infty}$ such that, for all $x^{0} \in \mathbb{R}^{n}$ and all $\Delta \in \mathcal{B}$, every global solution $x$ satisfies

$$
\|x(t)\| \leq \max \left\{\beta_{1}\left(t,\left\|x^{0}\right\|\right), \beta_{2}\left(\|\Delta\|_{L^{\infty}[0, t]}+c\right)\right\} \quad \forall t \in \mathbb{R}_{+} .
$$

System (2.1) is input-to-state stable if it is input-to-state stable with bias 0.

System (2.1) has the converging-input-converging-state property if, for all $x^{0} \in \mathbb{R}^{n}$ and all $\Delta \in \mathcal{B}$ with $\|\Delta\|_{L^{\infty}[t, \infty)} \rightarrow 0$ as $t \rightarrow \infty$, every maximal solution $x$ of (2.1) is global and satisfies $x(t) \rightarrow 0$ as $t \rightarrow \infty$. The following lemma shows in particular that if system (2.1) is input-to-state stable, then it has the converginginput-converging-state property.

LEMma 3.3. Assume that system (2.1) is input-to-state stable with bias $c \geq 0$ and let $\beta_{1}$ and $\beta_{2}$ be as in Definition 3.2. Let $x^{0} \in \mathbb{R}^{n}$ and $\Delta \in \mathcal{B}$. If $\Delta$ is essentially bounded $\left(\|\Delta\|_{L^{\infty}[0, \infty)}<\infty\right)$, then every global solution $x$ of (2.1) satisfies

$$
\limsup _{t \rightarrow \infty}\|x(t)\| \leq \limsup _{t \rightarrow \infty} \beta_{2}\left(\|\Delta\|_{L^{\infty}[t, \infty)}+c\right) .
$$

Proof. Let $x^{0} \in \mathbb{R}^{n}$ and let $\Delta \in \mathcal{B}$ be essentially bounded. Let $x$ be a global solution of (2.1), let $\tau \geq 0$ be arbitrary and set $x_{\tau}(t):=x(t+\tau)$ and $\Delta_{\tau}(t):=$ $\Delta(t+\tau)$ for all $t \geq 0$. Then, $\Delta_{\tau} \in \mathcal{B}$ and $x_{\tau}$ satisfies the initial-value problem

$$
\dot{x}_{\tau}(t)-A x_{\tau}(t) \in B\left(\Delta_{\tau}(t)-\Phi\left(C x_{\tau}(t)\right)\right), \quad x_{\tau}(0)=x(\tau) .
$$

By input-to-state stability with bias $c$,

$$
\begin{aligned}
\|x(t+\tau)\|=\left\|x_{\tau}(t)\right\| & \leq \max \left\{\beta_{1}(t,\|x(\tau)\|), \beta_{2}\left(\left\|\Delta_{\tau}\right\|_{L^{\infty}[0, t]}+c\right)\right\} \\
& =\max \left\{\beta_{1}(t,\|x(\tau)\|), \beta_{2}\left(\|\Delta\|_{L^{\infty}[\tau, t+\tau]}+c\right)\right\} \quad \forall t \in \mathbb{R}_{+} .
\end{aligned}
$$

Therefore, $\lim \sup _{t \rightarrow \infty}\|x(t)\| \leq \beta_{2}\left(\|\Delta\|_{L^{\infty}[\tau, \infty)}+c\right)$ for all $\tau \geq 0$, from which the claim follows.

We now state the two main results on input-to-state stability. The proofs can be found in Sections 4 and 5 .

Theorem 3.4. Let the linear system $(A, B, C)$ be stabilizable and detectable. Assume that (H1) holds. Then, every maximal solution of (2.1) is global and there exist positive constants $c_{1}, c_{2}$ and $\varepsilon$ such that, for all $x^{0} \in \mathbb{R}^{n}$ and $\Delta \in \mathcal{B}$, every global solution $x$ satisfies

$$
\|x(t)\| \leq c_{1} e^{-\varepsilon t}\left\|x^{0}\right\|+c_{2}\|\Delta\|_{L^{\infty}[0, t]} \quad \forall t \in \mathbb{R}_{+} .
$$

In particular, system (2.1) is input-to-state stable.

TheOREm 3.5. Let the linear system $(A, B, C)$ be minimal. Assume that at least one of hypotheses $(\mathrm{H} 2),(\mathrm{H} 3)$ or $(\mathrm{H} 4)$ holds. Then system $(2.1)$ is input-to-state stable.

In [1] it is has been proved, for single-valued $\Phi$ and $\Delta$, that, if (H4) holds, then (2.1) is input-to-state stable. Therefore, Theorem 3.5 can be considered as a generalization of the main result in [1]. 
In the following two corollaries (to Theorem 3.4 and Theorem 3.5, respectively) we will consider not only nonlinearities satisfying at least one of the conditions (3.1), (3.2), (3.4) and (3.5) for all arguments $y \in \mathbb{R}^{m}$, but also nonlinearities $\Phi \in \mathcal{U}$ with the property that there exist a set-valued map $\tilde{\Phi} \in \mathcal{U}$ satisfying at least one of the conditions (3.1), (3.2), (3.4) and (3.5) and a compact set $K \subset \mathbb{R}^{m}$ such that

$$
y \in \mathbb{R}^{m} \backslash K \quad \Longrightarrow \quad \Phi(y) \subset \tilde{\Phi}(y) .
$$

For example, single-input, single-output hysteretic elements can be subsumed by this set-valued formulation provided that the "characteristic diagram" of the hysteresis is contained in the graph of some $\Phi \in \mathcal{U}$, see Section 6 for details.

Corollary 3.6. Let the linear system $(A, B, C)$ be stabilizable and detectable. Let $\Phi \in \mathcal{U}$ be such that there exist a set-valued map $\tilde{\Phi} \in \mathcal{U}$ and a compact set $K \subset \mathbb{R}^{m}$ such that (3.9) holds. Assume that (H1) holds with $\Phi$ replaced by $\tilde{\Phi}$. Then, every maximal solution of (2.1) is global and there exist positive constants $c_{1}, c_{2}$ and $\varepsilon$ such that, for all $x^{0} \in \mathbb{R}^{n}$ and $\Delta \in \mathcal{B}$, every global solution $x$ satisfies

$$
\|x(t)\| \leq c_{1} e^{-\varepsilon t}\left\|x^{0}\right\|+c_{2}\left(\|\Delta\|_{L^{\infty}[0, t]}+E\right) \quad \forall t \in \mathbb{R}_{+},
$$

where

$$
E:=\sup _{y \in K} \sup _{v \in \Phi(y)} \inf _{\tilde{v} \in \tilde{\Phi}(y)}\|v-\tilde{v}\| .
$$

Proof. First, we remark that, by upper semicontinuity of $\Phi$ and $\tilde{\Phi} \in \mathcal{U}$, together with compactness of their values and compactness of $K, E$ is finite. Let $x^{0} \in \mathbb{R}^{n}$ and $\Delta \in \mathcal{B}$. By Lemma 2.2, (2.1) has a solution and every solution can be maximally extended. Let $x:[0, \omega) \rightarrow \mathbb{R}^{n}$ be a maximal solution of $(2.1)$ and write $y:=C x$. Define $z \in L_{\text {loc }}^{1}\left([0, \omega), \mathbb{R}^{n}\right)$ by $z:=\dot{x}-A x$. Since $z(t) \in B(\Delta(t)-\Phi(C x(t)))$ for almost every $t \in[0, \omega)$, there exist functions $d, v:[0, \omega) \rightarrow \mathbb{R}^{m}$ such that

$(d(t), v(t)) \in \Delta(t) \times \Phi(y(t)) \quad \forall t \in[0, \omega), \quad z(t)=B(d(t)-v(t))$ for a.e. $t \in[0, \omega)$.

For each $t \in[0, \omega)$, let $\tilde{v}(t) \in \tilde{\Phi}(y(t))$ be the unique point of the closed convex set $\tilde{\Phi}(y(t))$ closest to $v(t) \in \Phi(y(t))$. Then

$$
y(t) \in K \quad \Longrightarrow \quad\|v(t)-\tilde{v}(t)\| \leq E, \quad y(t) \in \mathbb{R}^{m} \backslash K \quad \Longrightarrow \quad\|v(t)-\tilde{v}(t)\|=0 .
$$

Define $\tilde{\Delta} \in \mathcal{B}$ by $\tilde{\Delta}(t):=\Delta(t)+\mathbb{B}_{E}$ (where $\mathbb{B}_{E}$ denotes the ball of radius $E>0$ centred at 0 in $\mathbb{R}^{m}$ ) and $\tilde{d}:[0, \omega) \rightarrow \mathbb{R}^{m}$ by $\tilde{d}(t):=d(t)-v(t)+\tilde{v}(t)$. Then

$$
z(t)=B(\tilde{d}(t)-\tilde{v}(t)), \quad \tilde{d}(t) \in \tilde{\Delta}, \quad \tilde{v}(t) \in \tilde{\Phi}(y(t)) \text { for a.e. } t \in[0, \omega)
$$

and so the solution $x$ of $(2.1)$ is also a solution of

$$
\dot{x}(t)-A x(t) \in B(\tilde{\Delta}(t)-\tilde{\Phi}(C x(t))), \quad x(0)=x^{0} .
$$

An application of Theorem 3.4 to (3.11) yields the claim.

Corollary 3.7. Let the linear system $(A, B, C)$ be minimal and let $\Phi \in \mathcal{U}$ be such that there exist a set-valued map $\tilde{\Phi} \in \mathcal{U}$ and a compact set $K \subset \mathbb{R}^{m}$ such that (3.9) holds. Assume that at least one of the hypotheses (H2), (H3) or (H4) holds with $\Phi$ replaced by $\tilde{\Phi}$. Then system (2.1) is input-to-state stable with bias $E$, where the constant $E$ is given by (3.10).

Proof. The proof is identical to that of Corollary 3.6 with one exception: instead of invoking Theorem 3.4 at the end of the proof, an application of Theorem 3.5 to (3.11) completes the argument here.

REMARK 3.8. If the hypotheses of Corollary 3.6 (respectively, Corollary 3.7) hold, then there exist positive constants $c_{1}, c_{2}, \varepsilon$ (respectively, functions $\beta_{1} \in \mathcal{K} \mathcal{L}$ and $\left.\beta_{2} \in \mathcal{K}_{\infty}\right)$ such that $(3.8)$ holds with $c=E$ given by (3.10). We emphasize that $c_{1}, c_{2}, \varepsilon$ (respectively, $\beta_{1}$ and $\beta_{2}$ ) are determined by data associated with only $(A, B, C)$ and $\tilde{\Phi}$ : in particular, they do not depend on $\Phi$. This observation is of importance in the analysis of quantized feedback systems in Section 7. 
4. Proof of Theorem 3.4. The following lemma will play an essential role in the proof of Theorem 3.4

LEMMA 4.1. Let $a<b$ and set $\kappa:=(a+b) / 2$ and $\lambda:=(b-a) / 2$. If $G(I+$ $a G)^{-1} \in H^{\infty}$ and there exists $\delta>0$ such that $(I+b G)(I+a G)^{-1}-\delta I$ is positive real, then $G(I+\kappa G)^{-1} \in H^{\infty}$ and $\left\|G(I+\kappa G)^{-1}\right\|_{H^{\infty}}<1 / \lambda$.

Proof. Setting $\eta:=\left\|G(I+a G)^{-1}\right\|_{H^{\infty}}$, we have that

$$
\left(I+a G^{*}(s)\right)^{-1} G^{*}(s) G(I+a G(s))^{-1} \leq \eta^{2} I \quad \forall s \in \mathbb{C}_{+} .
$$

By hypothesis,

$$
(I+b G(s))(I+a G(s))^{-1}+\left(I+a G^{*}(s)\right)^{-1}\left(I+b G^{*}(s)\right) \geq 2 \delta I \quad \forall s \in \mathbb{C}_{+} .
$$

Setting $\varepsilon:=\delta / \eta^{2}$, we obtain that

$$
\begin{aligned}
& 2 \varepsilon\left(I+a G^{*}(s)\right)^{-1} G^{*}(s) G(s)(I+a G(s))^{-1} \\
& \quad \leq(I+b G(s))(I+a G(s))^{-1}+\left(I+a G^{*}(s)\right)^{-1}\left(I+b G^{*}(s)\right) \quad \forall s \in \mathbb{C}_{+} .
\end{aligned}
$$

Therefore,

$$
2 \varepsilon G^{*}(s) G(s) \leq 2 I+(a+b) G^{*}(s)+(a+b) G(s)+2 a b G^{*}(s) G(s) \quad \forall s \in \Gamma,
$$

where $\Gamma:=\left\{s \in \mathbb{C}_{+} \mid s\right.$ not a pole of $\left.G\right\}$. Consequently,

$$
-(a b-\varepsilon) G^{*}(s) G(s) \leq I+\kappa G^{*}(s)+\kappa G(s) \quad \forall s \in \Gamma .
$$

Setting $\rho:=\sqrt{1+\varepsilon / \lambda^{2}}$, it follows that

$$
\begin{aligned}
\lambda^{2} \rho^{2} G^{*}(s) G(s) \leq I+\kappa G^{*}(s)+\kappa G(s)+ & \kappa^{2} G^{*}(s) G(s) \\
& =\left(I+\kappa G^{*}(s)\right)(I+\kappa G(s)) \quad \forall s \in \Gamma,
\end{aligned}
$$

which in turn implies that

$$
\rho^{2}\left(I+\kappa G^{*}(s)\right)^{-1} G^{*}(s) G(s)(I+\kappa G(s))^{-1} \leq \lambda^{-2} I \quad \forall s \in \Gamma_{0},
$$

where $\Gamma_{0}:=\{s \in \Gamma \mid \operatorname{det}(s I+\kappa G(s)) \neq 0\}$. We may now infer that $G(I+\kappa G)^{-1} \in$ $H^{\infty}$ and, since $\rho>1,\left\|G(I+\kappa G)^{-1}\right\|_{H^{\infty}}<1 / \lambda$. $\square$

Proof of Theorem 3.4. Let $x$ be a maximal solution of (2.1) defined on the maximal interval of existence $[0, \omega)$, where $0<\omega \leq \infty$. We first show that $\omega=\infty$. Seeking a contradiction, suppose that $\omega<\infty$. A routine application of the generalized Filippov selection theorem (see [22], p. 72) shows that there exists a measurable function $w:[0, \omega) \rightarrow \mathbb{R}^{m}$ such that $w(t) \in \Delta(t)-\Phi(C x(t))$ for a.e. $t \in[0, \omega)$ and

$$
\dot{x}(t)=A x(t)+B w(t) \quad \text { a.e. } t \in[0, \omega) .
$$

Setting $\kappa:=(a+b) / 2$ and $A_{\kappa}:=A-\kappa B C$, we have

$$
\left.x(t)=e^{A_{\kappa} t} x^{0}+\int_{0}^{t} e^{A_{\kappa}(t-\tau)} B(w(\tau)+\kappa C x(\tau))\right) \mathrm{d} \tau \quad \forall t \in[0, \omega) .
$$

Since $w(t) \in \Delta(t)-\Phi(C x(t))$ for a.e. $t \in[0, \omega)$, there exist functions $d, v:[0, \omega) \rightarrow$ $\mathbb{R}^{m}$ (not necessarily measurable) such that $w(t)=d(t)-v(t), d(t) \in \Delta(t)$ and $v(t) \in \Phi(C x(t))$ for a.e. $t \in[0, \omega)$. Setting $\lambda:=(b-a) / 2$ and invoking the sector condition (3.1) combined with part (a) of Remark 3.1, we may infer that

$$
\begin{aligned}
& \|w(\tau)+\kappa C x(\tau)\|=\|d(\tau)-(v(\tau)-\kappa C x(\tau))\| \\
& \quad \leq\|d(\tau)\|+\|(v(\tau)-\kappa C x(\tau))\| \leq|\Delta(\tau)|+\lambda\|C x(\tau)\| \text { for a.e. } \tau \in[0, \omega) .
\end{aligned}
$$


Therefore,

$$
\begin{aligned}
\|x(t)\| \leq\left\|e^{A_{\kappa} t} x^{0}\right\|+\|B\| \int_{0}^{t} & \left\|e^{A_{\kappa}(t-\tau)}\right\| \| \Delta(\tau) \mid \mathrm{d} \tau \\
& +\lambda\|B\|\|C\| \int_{0}^{t}\left\|e^{A_{\kappa}(t-\tau)}\right\|\|x(\tau)\| \mathrm{d} \tau \quad \forall t \in[0, \omega) .
\end{aligned}
$$

Since (by supposition) $\omega$ is finite, we conclude that, for some constant $c>0$,

$$
\|x(t)\| \leq c\left(1+\int_{0}^{t}\|x(\tau)\| \mathrm{d} \tau\right) \quad \forall t \in[0, \omega) .
$$

By Gronwall's lemma, it follows that the maximal solution $x$ is bounded on $[0, \omega)$, contradicting (via Lemma 2.2) the supposition that $\omega<\infty$. Consequently, $\omega=\infty$.

Defining $G_{\kappa}(s):=G(I+\kappa G(s))^{-1}=C\left(s I-A_{\kappa}\right)^{-1} B$, it follows from (H1), via Lemma 4.1, that $G_{\kappa} \in H^{\infty}$ and $\left\|G_{\kappa}\right\|_{H^{\infty}}<1 / \lambda$. Moreover, by stabilizability and detectability, $A_{\kappa}$ is Hurwitz. Let $\varepsilon>0$ be sufficiently small so that $A_{\kappa}+\varepsilon I$ is Hurwitz and

$$
\gamma:=\sup _{\operatorname{Re} s \geq-\varepsilon}\left\|G_{\kappa}(s)\right\|<1 / \lambda .
$$

Set $y:=C x$ and, for all $t \in \mathbb{R}_{+}$, define $y_{\varepsilon}(t):=e^{\varepsilon t} y(t)$ and $w_{\varepsilon}(t):=e^{\varepsilon t} w(t)$. It follows from (4.1) that

$$
\left.y_{\varepsilon}(t)=C e^{\left(A_{\kappa}+\varepsilon I\right) t} x^{0}+\int_{0}^{t} C e^{\left(A_{\kappa}+\varepsilon I\right)(t-\tau)} B\left(w_{\varepsilon}(\tau)+\kappa y_{\varepsilon}(\tau)\right)\right) \mathrm{d} \tau \quad \forall t \in \mathbb{R}_{+} .
$$

Setting $k_{0}:=\left(\int_{0}^{\infty}\left\|C e^{\left(A_{\kappa}+\varepsilon I\right) \tau}\right\|^{2} \mathrm{~d} \tau\right)^{1 / 2}<\infty$, we obtain that

$$
\left\|y_{\varepsilon}\right\|_{L^{2}[0, t]} \leq k_{0}\left\|x^{0}\right\|+\gamma\left\|w_{\varepsilon}+\kappa y_{\varepsilon}\right\|_{L^{2}[0, t]} \quad \forall t \in \mathbb{R}_{+} .
$$

By (4.2),

$$
\left.\left.\| w_{\varepsilon}(\tau)+\kappa y_{\varepsilon}(\tau)\right)\left\|\leq\left|\Delta_{\varepsilon}(\tau)\right|+\lambda\right\| y_{\varepsilon}(\tau)\right) \| \text { for a.e. } \tau \in \mathbb{R}_{+},
$$

where $\Delta_{\varepsilon}(\tau):=e^{\varepsilon \tau} \Delta(\tau)$ for all $\tau \in \mathbb{R}_{+}$. From (4.3), we see that $\gamma \lambda<1$ : setting $k_{1}:=1 /(1-\gamma \lambda)$ and invoking (4.4) and (4.5), we have

$$
\left\|y_{\varepsilon}\right\|_{L^{2}[0, t]} \leq k_{1}\left(k_{0}\left\|x^{0}\right\|+\gamma\left\|\Delta_{\varepsilon}\right\|_{L^{2}[0, t]}\right) \quad \forall t \in \mathbb{R}_{+} .
$$

By (4.1),

$$
\left.e^{\varepsilon t} x(t)=e^{\left(A_{\kappa}+\varepsilon I\right) t} x^{0}+\int_{0}^{t} e^{\left(A_{\kappa}+\varepsilon I\right)(t-\tau)} B\left(w_{\varepsilon}(\tau)+\kappa y_{\varepsilon}(\tau)\right)\right) \mathrm{d} \tau \quad \forall t \in \mathbb{R}_{+},
$$

which, together with (4.5), yields

$$
\|x(t)\| e^{\varepsilon t} \leq k_{2}\left\|x^{0}\right\|+\|B\| \int_{0}^{t}\left\|e^{\left(A_{\kappa}+\varepsilon I\right)(t-\tau)}\right\|\left(\left|\Delta_{\varepsilon}(\tau)\right|+\lambda\left\|y_{\varepsilon}(\tau)\right\|\right) \mathrm{d} \tau \quad \forall t \in \mathbb{R}_{+},
$$

where $k_{2}:=\sup _{t \geq 0}\left\|e^{\left(A_{\kappa}+\varepsilon I\right) t}\right\|$. Invoking Hölder's inequality to estimate the integral on the right-hand side of the above inequality, we conclude that there exists a constant $k_{3}>0$ such that

$$
\|x(t)\| e^{\varepsilon t} \leq k_{2}\left\|x^{0}\right\|+k_{3}\left(\left\|\Delta_{\varepsilon}\right\|_{L^{2}[0, t]}+\lambda\left\|y_{\varepsilon}\right\|_{L^{2}[0, t]}\right) \quad \forall t \in \mathbb{R}_{+} .
$$


Combining this with (4.6), we conclude that,

$$
\|x(t)\| e^{\varepsilon t} \leq\left(k_{2}+\lambda k_{0} k_{1} k_{3}\right)\left\|x^{0}\right\|+k_{3}\left(1+\lambda \gamma k_{1}\right)\left\|\Delta_{\varepsilon}\right\|_{L^{2}[0, t]} \quad \forall t \in \mathbb{R}_{+} .
$$

Noting that $\left\|\Delta_{\varepsilon}\right\|_{L^{2}[0, t]} \leq\left(e^{\varepsilon t} / \sqrt{2 \varepsilon}\right)\|\Delta\|_{L^{\infty}[0, t]}$ for all $t \in \mathbb{R}_{+}$, setting $c_{1}:=k_{2}+$ $\lambda k_{0} k_{1} k_{3}$ and $c_{2}:=k_{3}\left(1+\lambda \gamma k_{1}\right) / \sqrt{2 \varepsilon}$, we conclude that

$$
\|x(t)\| \leq c_{1} e^{-\varepsilon t}\left\|x^{0}\right\|+c_{2}\|\Delta\|_{L^{\infty}[0, t]} \quad \forall t \in \mathbb{R}_{+},
$$

This completes the proof.

REMARK 4.2. Theorem 3.4 can be considered as a refinement of the classical circle criterion (see, for example, $[7,13,21])$ : in particular, it shows that, under the standard assumptions of the circle criterion, input-to-state stability is guaranteed. The exponential weighting technique used in the proof of Theorem 3.4 is well-known and has been used to prove stability results of input-output type (see [7, Section V.3] and the references therein). The application of this technique in an input-tostate stability context seems to be new. In particular, whilst the standard textbook version of the circle criterion for state-space systems is usually proved using Lyapunov techniques combined with the Positive-Real Lemma (see, for example, [13, Theorem 7.1] or [21, p. 227]), the above proof of Theorem 3.4 provides an alternative, more elementary, approach. Moreover, the methodology can be extended to an infinite-dimensional setting: see [11].

5. Proof of Theorem 3.5. In this section, we provide a proof of Theorem 3.5. In contrast to the proof of Theorem 3.4, we adopt a Lyapunov argument. In particular, we prove Theorem 3.5 by establishing the existence of a Lyapunov function with special properties (a so-called ISS Lyapunov function) if any one of hypotheses (H2), (H3) or (H4) hold. This we do in two preliminary lemmas.

Lemma 5.1. Let the linear system $(A, B, C)$ be minimal. Assume that either (H3) or (H4) holds. Then there exist $\alpha_{1}, \alpha_{2}, \alpha_{3}, \alpha_{4} \in \mathcal{K}_{\infty}$ and a continuously differentiable function $V: \mathbb{R}^{n} \rightarrow \mathbb{R}_{+}$such that:

$$
\left.\begin{array}{rl}
\alpha_{1}(\|x\|) \leq V(x) \leq \alpha_{2}(\|x\|) \quad \forall x \in \mathbb{R}^{n}, & \\
\max _{v \in \Phi(C x)}\langle\nabla V(x), A x+B(d-v)\rangle \leq-\alpha_{3}(\|x\|)+\alpha_{4}(\|d\|) \\
\forall(x, d) \in \mathbb{R}^{n} \times \mathbb{R}^{m} .
\end{array}\right\}
$$

Proof. By Lemma 2.1, there exists $\psi \in \mathcal{K}_{\infty}$ such that

$$
\|v\| \leq \psi(\|y\|) \quad \forall y \in \mathbb{R}^{m}, \quad \forall v \in \Phi(y) .
$$

(If (H3) holds, then we may take $\psi: s \mapsto b s$ in (5.2).) Combining (5.2) with either (H3) or (H4) yields

$$
\varphi(\|y\|)\|y\| \leq\langle y, v\rangle \leq \psi(\|y\|)\|y\| \quad \forall y \in \mathbb{R}^{m}, \quad \forall v \in \Phi(y) .
$$

If (H3) holds, then $(\delta / b) I+G$ is positive real for some $\delta \in[0,1)$; if (H4) holds then $G$ is positive real. Introducing the following notational convenience

$$
\lambda:=\left\{\begin{array}{cl}
1 / b & \text { if }(\mathrm{H} 3) \text { holds } \\
0 & \text { otherwise }
\end{array}\right.
$$

both possibilities are captured by the statement that $\delta \lambda I+G$ is positive real for some $\delta \in[0,1)$. This implies, via by the Positive-Real Lemma, the existence of a real matrix $L$ and a symmetric, positive-definite real matrix $P$ such that

$$
P A+A^{T} P=-L^{T} L, \quad P B=C^{T}-\sqrt{\kappa} L^{T}, \quad \kappa:=2 \delta \lambda .
$$


We also record that

$$
\lambda\|v\|^{2} \leq\langle y, v\rangle \quad \forall v \in \Phi(y), \quad \forall y \in \mathbb{R}^{m} .
$$

Now, define $V_{0}: \mathbb{R}^{n} \rightarrow \mathbb{R}_{+}, x \mapsto\langle x, P x\rangle$. Then, invoking (5.4),

$$
\begin{aligned}
&\left\langle\nabla V_{0}(x), A x+B(d-v)\right\rangle=2\langle P x, A x\rangle+2\left\langle B^{T} P x,(d-v)\right\rangle \\
& \leq-\|L x\|^{2}+2\langle C x,(d-v)\rangle-2 \sqrt{\kappa}\langle L x,(d-v)\rangle \\
&=-\|L x+\sqrt{\kappa}(d-v)\|^{2}+\kappa\|d-v\|^{2}+2\langle C x,(d-v)\rangle \\
& \forall x \in \mathbb{R}^{n}, \quad \forall(d, v) \in \mathbb{R}^{m} \times \Phi(C x),
\end{aligned}
$$

from which, together with (5.5), we may infer

$$
\begin{gathered}
\left\langle\nabla V_{0}(x), A x+B(d-v)\right\rangle \leq \kappa\|d\|^{2}+2 \kappa\|v\|\|d\|+\kappa\|v\|^{2}+2\|y\|\|d\|-2\langle y, v\rangle \\
\leq 2(1+2 \delta)\|y\|\|d\|+\kappa\|d\|^{2}-2(1-\delta)\langle y, v\rangle \\
\forall x \in \mathbb{R}^{n}, \quad \forall(d, v) \in \mathbb{R}^{m} \times \Phi(y), y=C x .
\end{gathered}
$$

Observe that, for all $y \in \mathbb{R}^{m}$ and all $(d, v) \in \mathbb{R}^{m} \times \Phi(y)$,

$$
\begin{gathered}
2(1+2 \delta)\|d\| \leq(1-\delta) \varphi(\|y\|) \Longrightarrow \\
2(1+2 \delta)\|d\|\|y\| \leq(1-\delta) \varphi(\|y\|)\|y\| \leq(1-\delta)\langle y, v\rangle \\
2(1+2 \delta)\|d\|>(1-\delta) \varphi(\|y\|) \Longrightarrow \\
2(1+2 \delta)\|d\|\|y\|<2(1+2 \delta)\|d\| \varphi^{-1}(2(1+2 \delta)\|d\| /(1-\delta))
\end{gathered}
$$

and so, defining $\gamma \in \mathcal{K}_{\infty}$ by $\gamma(s):=2(1+2 \delta) s \varphi^{-1}(2(1+2 \delta) s /(1-\delta))$, we have

$$
2(1+2 \delta)\|d\|\|y\| \leq(1-\delta)\langle y, v\rangle+\gamma(\|d\|) \quad \forall y \in \mathbb{R}^{m}, \quad \forall(d, v) \in \mathbb{R}^{m} \times \Phi(y) .
$$

The conjunction of (5.6) and (5.7) gives

$$
\begin{aligned}
&\left\langle\nabla V_{0}(x), A x+B(d-v)\right\rangle \leq-(1-\delta)\langle y, v\rangle+\gamma(\|d\|)+\kappa\|d\|^{2} \\
& \forall x \in \mathbb{R}^{n}, \forall(d, v) \in \mathbb{R}^{m} \times \Phi(y), y=C x .
\end{aligned}
$$

Let $H \in \mathbb{R}^{n \times m}$ be such that $A-H C$ is Hurwitz. Let $Q=Q^{T}>0$ be such that

$$
Q(A-H C)+(A-H C)^{T} Q=-3 I,
$$

and define $W: \mathbb{R}^{n} \rightarrow \mathbb{R}_{+}$by $W(x):=\langle x, Q x\rangle$.

Writing $k_{0}:=\max \left\{2\|Q B\|, 2\|Q H\|,\|Q B\|^{2}\right\}$, we have

$$
\begin{aligned}
\langle\nabla W(x), A x+B(d-v)\rangle & =2\langle Q x,(A-H C) x+H y+B(d-v)\rangle \\
& =-3\|x\|^{2}+2\left\langle H^{T} Q x, y\right\rangle+2\left\langle B^{T} Q x, d-v\right\rangle \\
\leq & -2\|x\|^{2}+k_{0}\|x\|(\|y\|+\|v\|)+k_{0}\|d\|^{2} \\
& \forall x \in \mathbb{R}^{n}, \forall(d, v) \in \mathbb{R}^{m} \times \Phi(y), y=C x .
\end{aligned}
$$

Since either (H3) or (H4) holds, and invoking part (f) of Remark 3.1 in the former case, we may infer the existence of $\theta \geq 1 / 2$ such that

$$
y \in \mathbb{R}^{m}, \quad\|y\| \geq \theta \quad \Longrightarrow \quad\langle y, v\rangle \geq\|v\| \quad \forall v \in \Phi(y) .
$$

Define $f_{0} \in \mathcal{K}_{\infty}$ by $f_{0}(s):=s+\psi(s)$, and the continuous, non-decreasing function $f_{1}:(0, \theta] \rightarrow(0, \infty)$ by

$$
f_{1}(s):=\min _{t \in[s, \theta]} \frac{t \varphi(t)}{\left(f_{0}(t)\right)^{2}}
$$


and $f_{2}: \mathbb{R}_{+} \rightarrow \mathbb{R}_{+}$by

$$
f_{2}(s):= \begin{cases}0, & s=0 \\ \min \left\{s, f_{1}(s)\right\}, & s \in(0, \theta] \\ f_{1}(\theta)+(s-\theta), & s>\theta\end{cases}
$$

Observe that

$$
f_{1}(\theta)=\frac{\theta \varphi(\theta)}{(\theta+\psi(\theta))^{2}}<\frac{\theta \varphi(\theta)}{2 \theta \psi(\theta)} \leq \frac{\theta \varphi(\theta)}{\psi(\theta)} \leq \theta,
$$

where we have used that $\theta \geq 1 / 2$. It follows that $f_{2}(\theta)=f_{1}(\theta)$, and therefore, $f_{2}$ is continuous. Clearly, $f_{2}$ is unbounded and, moreover, it is readily verified that $f_{2}$ is non-decreasing. Write $f_{3}:=f_{2} \circ f_{0}^{-1}$ (continuous, non-decreasing and unbounded, with $f_{3}(0)=0$ ) and observe (for later use) that

$$
\begin{gathered}
\|y\|<\theta \Longrightarrow \quad f_{3}(\|y\|+\psi(\|y\|))(\|y\|+\psi(\|y\|))^{2}=\left(f_{3} \circ f_{0}\right)(\|y\|)\left(f_{0}(\|y\|)\right)^{2} \\
=f_{2}(\|y\|)\left(f_{0}(\|y\|)\right)^{2} \leq f_{1}(\|y\|)\left(f_{0}(\|y\|)\right)^{2} \leq\|y\| \varphi(\|y\|) .
\end{gathered}
$$

Next, we introduce functions $\eta \in \mathcal{K}_{\infty}$ and $\sigma$ (continuous, non-decreasing and unbounded, with $\sigma(0)=0$ ) given by

$$
\eta: s \mapsto \frac{1}{k_{0}} \sqrt{\frac{s}{\|Q\|}}, \quad \sigma:=f_{3} \circ \eta .
$$

Let $s^{*}>0$ be the unique point with the property $\eta\left(s^{*}\right) \sigma\left(s^{*}\right)=1$ and define the continuous function $\rho: \mathbb{R}_{+} \rightarrow \mathbb{R}_{+}$by

$$
\rho(s):=\left\{\begin{array}{l}
\sigma(s), \quad 0 \leq s \leq s^{*} \\
1 / \eta(s), \quad s>s^{*}
\end{array}\right.
$$

Finally, define $R \in \mathcal{K}_{\infty}$ by

$$
R(s):=\int_{0}^{s} \rho(\tau) d \tau
$$

and $V_{1}: \mathbb{R}^{n} \rightarrow \mathbb{R}_{+}, \quad x \mapsto R(W(x))$. Note that

$$
\left.\begin{array}{l}
\text { (a) } \rho(s) \leq \sigma(s) \leq \sigma\left(s^{*}\right)=: k_{1} \quad \forall s \in \mathbb{R}_{+}, \\
\text {(b) } \rho(W(x))\|x\| \leq k_{0} \sqrt{\|Q\|\left\|Q^{-1}\right\|}=: k_{2} \quad \forall x \in \mathbb{R}^{n}, \\
\text { (c) } \left.\rho(W(x))\|x\|^{2} \geq\|x\| \min \left\{\|x\| f_{3}\left(\|x\| / k_{2}\right)\right), k_{0}\right\} \quad \forall x \in \mathbb{R}^{n} .
\end{array}\right\}
$$

Invoking (5.9) and (5.12)(a), we have

$$
\begin{array}{r}
\left\langle\nabla V_{1}(x), A x+B(d-v)\right\rangle \leq-2 \rho(W(x))\|x\|^{2}+\rho(W(x)) k_{0}\|x\|(\|y\|+\|v\|)+k_{0} k_{1}\|d\|^{2} \\
\forall x \in \mathbb{R}^{n}, \forall(d, v) \in \mathbb{R}^{m} \times \Phi(C x) .
\end{array}
$$

We proceed to obtain a convenient estimate of the term $\rho(W(x)) k_{0}\|x\|(\|y\|+\|v\|)$.

Write $k_{3}:=\frac{1}{2} \min \{1, \varphi(\theta)\}$. By (5.3) and (5.10), we have

$$
\begin{array}{r}
\|y\| \geq \theta \Longrightarrow 2\langle y, v\rangle \geq\|v\|+\|y\| \varphi(\|y\|) \geq\|v\|+\|y\| \varphi(\theta) \geq 2 k_{3}(\|v\|+\|y\|) \\
\forall v \in \Phi(y)
\end{array}
$$

which, in conjunction with (5.12)(b), gives

$$
\begin{aligned}
& x \in \mathbb{R}^{n}, y=C x,\|y\| \geq \theta \Longrightarrow \\
& \rho(W(x)) k_{0}\|x\|(\|y\|+\|v\|) \leq \frac{k_{0} k_{2}}{k_{3}}\langle y, v\rangle \quad \forall v \in \Phi(y) .
\end{aligned}
$$


Invoking (5.2), (5.3) and (5.11), we have

$$
\begin{aligned}
\|y\|<\theta & \Longrightarrow f_{3}(\|y\|+\|v\|)(\|y\|+\|v\|)^{2} \\
& \leq f_{3}(\|y\|+\psi(\|y\|))(\|y\|+\psi(\|y\|))^{2} \leq\|y\| \varphi(\|y\|) \leq\langle y, v\rangle \quad \forall v \in \Phi(y)
\end{aligned}
$$

from which, together with the observation that

$$
\begin{aligned}
x \in \mathbb{R}^{n}, y & =C x, v \in \Phi(y), k_{0}(\|y\|+\|v\|) \geq\|x\| \Longrightarrow \\
& \left.(W(x)) \leq \sigma\left(\|Q\|\|x\|^{2}\right)\right) \leq \sigma\left(k_{0}^{2}\|Q\|(\|y\|+\|v\|)^{2}\right)=f_{3}(\|y\|+\|v\|),
\end{aligned}
$$

we may infer

$$
\begin{array}{r}
x \in \mathbb{R}^{n}, y=C x, v \in \Phi(y), k_{0}(\|y\|+\|v\|) \geq\|x\|,\|y\|<\theta \Longrightarrow \\
\rho(W(x)) k_{0}\|x\|(\|y\|+\|v\|) \leq \rho(W(x))\|x\|^{2}+\frac{k_{0}^{2}}{4} \rho(W(x))(\|y\|+\|v\|)^{2} \\
\leq \rho(W(x))\|x\|^{2}+\frac{k_{0}^{2}}{4}\langle y, v\rangle .
\end{array}
$$

Clearly,

$$
\begin{aligned}
x \in \mathbb{R}^{n}, y=C x, v \in \Phi(y), & k_{0}(\|y\|+\|v\|) \leq\|x\|,\|y\|<\theta \Longrightarrow \\
& \Longrightarrow(W(x)) k_{0}\|x\|(\|y\|+\|v\|) \leq \rho(W(x))\|x\|^{2} .
\end{aligned}
$$

Combining (5.15) and (5.16), we have

$$
\begin{aligned}
& x \in \mathbb{R}^{n}, y=C x,\|y\|<\theta \Longrightarrow \\
& \quad \rho(W(x)) k_{0}\|x\|(\|y\|+\|v\|) \leq \rho(W(x))\|x\|^{2}+\frac{k_{0}^{2}}{4}\langle y, v\rangle \quad \forall v \in \Phi(y) .
\end{aligned}
$$

Writing $k_{4}:=\max \left\{k_{0} k_{2} / k_{3}, k_{0}^{2} / 4\right\}$, we conclude, from (5.13), (5.14), (5.17), that

$$
\begin{array}{r}
\left\langle\nabla V_{1}(x), A x+B(d-v)\right\rangle \leq-\rho(W(x))\|x\|^{2}+k_{4}\langle y, v\rangle+k_{0} k_{1}\|d\|^{2} \\
\forall x \in \mathbb{R}^{n}, \forall(d, v) \in \mathbb{R}^{m} \times \Phi(C x) .
\end{array}
$$

Now define $V:=k_{4} V_{0}+(1-\delta) V_{1}$. Then, combining (5.8) and (5.18), we arrive at

$$
\begin{aligned}
& \langle\nabla V(x), A x+B(d-v)\rangle \\
& \leq-(1-\delta) \rho(W(x))\|x\|^{2}+\left((1-\delta) k_{0} k_{1}+\kappa k_{4}\right)\|d\|^{2}+k_{4} \gamma(\|d\|) \\
& \forall x \in \mathbb{R}^{n}, \quad \forall(d, v) \in \mathbb{R}^{m} \times \Phi(y), y=C x
\end{aligned}
$$

Finally, defining $\alpha_{1}, \alpha_{2}, \alpha_{3}, \alpha_{4} \in \mathcal{K}_{\infty}$ by

$$
\begin{aligned}
& \alpha_{1}(s):=k_{4}\left\|P^{-1}\right\|^{-1} s^{2}, \quad \alpha_{2}:=k_{4}\|P\| s^{2}+(1-\delta) R\left(\|Q\| s^{2}\right), \\
& \alpha_{3}(s):=(1-\delta) s \min \left\{s f_{3}\left(s / k_{2}\right), k_{0}\right\}, \quad \alpha_{4}(s):=\left((1-\delta) k_{0} k_{1}+\kappa k_{4}\right) s^{2}+k_{4} \gamma(s),
\end{aligned}
$$

and invoking (5.12)(c), we conclude that (5.1) holds. This completes the proof.

Lemma 5.2. Let the linear system $(A, B, C)$ be minimal. Assume that (H2) holds. Then the assertions of Lemma 5.1 are valid.

Proof. Let $a>0, \delta \in[0,1)$ and $\theta \geq 0$ be as in hypothesis (H2). Without loss of generality, we may assume $\theta \geq 1 / 2$. Note that the linear system $\left(A_{1}, B, C\right)$, with $A_{1}:=A-\delta a B C$, is a minimal realization of $G(I+\delta a G)^{-1}$. Therefore, hypothesis 
(H2) implies, via the Positive-Real Lemma, the existence of a real matrix $L$ and a symmetric, positive-definite real matrix $P$ such that

$$
P A_{1}+A_{1}^{T} P=-L^{T} L, \quad P B=C^{T} .
$$

Invoking Lemma 2.1, there exists $\psi \in \mathcal{K}_{\infty}$ such that (5.3) holds with $\varphi(s)=$ as. Now define $\varphi_{1}, \psi_{1} \in \mathcal{K}_{\infty}$ and $y \mapsto \Phi_{1}(y) \subset \mathbb{R}^{m}$ by

$$
\begin{gathered}
\varphi_{1}(s):=\varphi(s)-\delta a s=(1-\delta) a s, \quad \psi_{1}(s):=\psi(s)-\delta a s \quad \forall s \in \mathbb{R}_{+} . \\
\Phi_{1}(y):=\{v-\delta a y \mid v \in \Phi(y)\} \quad \forall y \in \mathbb{R}^{m} .
\end{gathered}
$$

In view of (5.3), we have

$$
(1-\delta) a\|y\|^{2}=\varphi_{1}(\|y\|)\|y\| \leq\langle y, v\rangle \leq \psi_{1}(\|y\|)\|y\| \quad \forall y \in \mathbb{R}^{m}, \quad \forall v \in \Phi_{1}(y) .
$$

Moreover, by hypothesis (H2),

$$
y \in \mathbb{R}^{m}, \quad\|y\| \geq \theta \quad \Longrightarrow \quad\langle y, v\rangle \geq\|v\| \quad \forall v \in \Phi_{1}(y) .
$$

Recalling that $A_{1}:=A-\delta a B C$, we have

$$
\{A x-B v \mid v \in \Phi(C x)\}=\left\{A_{1} x-B v \mid v \in \Phi_{1}(C x)\right\} \quad \forall x \in \mathbb{R}^{n} .
$$

Now, define $V_{0}: \mathbb{R}^{n} \rightarrow \mathbb{R}_{+}, x \mapsto\langle x, P x\rangle$. Then, invoking (5.20),

$$
\begin{aligned}
\left\langle\nabla V_{0}(x), A_{1} x+B(d-v)\right\rangle= & 2\left\langle P x, A_{1} x\right\rangle+2\left\langle B^{T} P x,(d-v)\right\rangle \\
\leq-\|L x\|^{2}+ & 2\langle C x,(d-v)\rangle \leq 2\|y\|\|d\|-2\langle y, v\rangle \\
& \forall x \in \mathbb{R}^{n}, \quad \forall(d, v) \in \mathbb{R}^{m} \times \Phi_{1}(y), y=C x,
\end{aligned}
$$

Observe that, for all $y \in \mathbb{R}^{m}$ and all $(d, v) \in \mathbb{R}^{m} \times \Phi_{1}(y)$,

$$
\begin{aligned}
& 2\|d\| \leq \varphi_{1}(\|y\|) \Longrightarrow 2\|d\|\|y\| \leq \varphi_{1}(\|y\|)\|y\| \leq\langle y, v\rangle \\
& 2\|d\|>\varphi_{1}(\|y\|) \Longrightarrow 2\|d\|\|y\|<2\|d\| \varphi_{1}^{-1}(2\|d\|)
\end{aligned}
$$

and so, defining $\gamma \in \mathcal{K}_{\infty}$ by $\gamma(s):=2 s \varphi_{1}^{-1}(2 s)$, it follows from (5.24) that

$$
\begin{aligned}
\left\langle\nabla V_{0}(x), A_{1} x+B(d-v)\right\rangle \leq & -\langle y, v\rangle+\gamma(\|d\|) \\
& \forall x \in \mathbb{R}^{n}, \forall(d, v) \in \mathbb{R}^{m} \times \Phi_{1}(y), y=C x .
\end{aligned}
$$

The conjunction of (5.23) and (5.25) yields

$$
\begin{aligned}
\left\langle\nabla V_{0}(x), A x+B(d-v)\right\rangle \leq-\langle y, v\rangle+\delta a\|y\|^{2}+\gamma(\|d\|) & \\
& \forall x \in \mathbb{R}^{n}, \forall(d, v) \in \mathbb{R}^{m} \times \Phi(y), y=C x .
\end{aligned}
$$

Let $H \in \mathbb{R}^{n \times m}$ be such that $A_{1}-H C$ is Hurwitz. Let $Q=Q^{T}>0$ be such that

$$
Q\left(A_{1}-H C\right)+\left(A_{1}-H C\right)^{T} Q=-3 I,
$$

and define $W: \mathbb{R}^{n} \rightarrow \mathbb{R}_{+}$by $W(x):=\langle x, Q x\rangle$. The same construction as in the proof of Lemma 5.1 (with $A_{1}$ replacing $A$ and $\Phi_{1}$ replacing $\Phi$ therein) yields a function $f_{3}$ (continuous, non-decreasing and unbounded, with $f_{3}(0)=0$ ), a continuous function $\rho: \mathbb{R}_{+} \rightarrow \mathbb{R}_{+}$, with primitive $R \in \mathcal{K}_{\infty}$, and positive constants $c_{0}, c_{1}, c_{2}, c_{3}$ 
such that, on writing $V_{1}: \mathbb{R}^{n} \rightarrow \mathbb{R}_{+}, x \mapsto R(W(x))$, the following counterparts of $(5.12)(\mathrm{c})$ and $(5.18)$ hold

$$
\begin{gathered}
\rho(W(x))\|x\|^{2} \geq\|x\| \min \left\{c_{0},\|x\| f_{3}\left(c_{1}\|x\|\right)\right\} \quad \forall x \in \mathbb{R}^{n}, \\
\left\langle\nabla V_{1}(x), A_{1} x+B(d-v)\right\rangle \leq-\rho(W(x))\|x\|^{2}+c_{2}\langle y, v\rangle+c_{3}\|d\|^{2} \\
\forall x \in \mathbb{R}^{n}, \forall(d, v) \in \mathbb{R}^{m} \times \Phi_{1}(y), y=C x .
\end{gathered}
$$

In view of (5.23), the latter yields

$$
\begin{array}{r}
\left\langle\nabla V_{1}(x), A x+B(d-v)\right\rangle \leq-\rho(W(x))\|x\|^{2}+c_{2}\langle y, v\rangle-c_{2} \delta a\|y\|^{2}+c_{3}\|d\|^{2} \\
\forall x \in \mathbb{R}^{n}, \forall(d, v) \in \mathbb{R}^{m} \times \Phi(y), y=C x,
\end{array}
$$

Now define $V:=c_{2} V_{0}+V_{1}$. Then, combining (5.26) and (5.28), we have

$$
\begin{aligned}
&\langle\nabla V(x), A x+B(d-v)\rangle \leq-\rho(W(x))\|x\|^{2}+c_{2} \gamma(\|d\|)+c_{3}\|d\|^{2} \\
& \forall x \in \mathbb{R}^{n}, \forall(d, v) \in \mathbb{R}^{m} \times \Phi(C x),
\end{aligned}
$$

Finally, defining $\alpha_{1}, \alpha_{2}, \alpha_{3}, \alpha_{4} \in \mathcal{K}_{\infty}$ by

$$
\begin{aligned}
& \alpha_{1}(s):=c_{2}\left\|P^{-1}\right\|^{-1} s^{2}, \quad \alpha_{2}:=c_{2}\|P\| s^{2}+R\left(\|Q\| s^{2}\right), \\
& \alpha_{3}(s):=s \min \left\{c_{0}, s f_{3}\left(c_{1} s\right)\right\}, \quad \alpha_{4}(s):=c_{2} \gamma(s)+c_{3} s^{2},
\end{aligned}
$$

and invoking (5.27), we may conclude that (5.1) holds. This completes the proof. $\square$

We are now in a position to prove Theorem 3.5. The argument developed below is not new and can be found (usually in form of sketch proofs) in the literature (see [20] and the references therein). For completeness we provide a detailed proof.

Proof of Theorem 3.5. If either (H3) or (H4) holds (respectively, if (H2) holds,) then Lemma 5.1 (respectively, Lemma 5.2) ensures the existence of $\alpha_{1}, \alpha_{2}, \alpha_{3}, \alpha_{4} \in$ $\mathcal{K}_{\infty}$ and continuously differentiable $V$ such that $\alpha_{1}(\|x\|) \leq V(x) \leq \alpha_{2}(\|x\|)$ for all $x \in \mathbb{R}^{n}$ and

$$
\begin{aligned}
&\langle\nabla V(x), A x+B(d-v)\rangle \leq-\alpha_{3}(\|x\|)+\alpha_{4}(\|d\|) \\
& \\
& \forall x \in \mathbb{R}^{n}, \quad \forall(d, v) \in \mathbb{R}^{m} \times \Phi(C x) .
\end{aligned}
$$

Let $x^{0} \in \mathbb{R}^{n}$ and $\Delta \in \mathcal{B}$. By Lemma 2.2, (2.1) has a solution and every solution can be maximally extended. Let $x:[0, \omega) \rightarrow \mathbb{R}^{n}$ be a maximal solution of (2.1). By (5.30), we have

$$
(V \circ x)^{\prime}(t) \leq \alpha_{4}(|\Delta(t)|) \quad \text { for a.e. } t \in[0, \omega) .
$$

Seeking a contradiction, suppose that $\omega<\infty$. Then, by local essential boundedness of $\Delta$ and continuity of $\alpha_{4}$, there exists $c_{0}>0$ such that $\alpha_{4}(|\Delta(t)|) \leq c_{0}$ for all $t \in$ $[0, \omega)$. Now, by the final assertion of Lemma $2.2, x$ is unbounded which contradicts the fact that, by $(5.31), \alpha_{1}(\|x(t)\|) \leq V(x(t)) \leq V\left(x^{0}\right)+c_{0} \omega$ for all $t \in[0, \omega)$. Therefore, every maximal solution of (2.1) is global.

Write $\alpha_{5}:=\alpha_{3} \circ \alpha_{2}^{-1} \in \mathcal{K}_{\infty}$ and define $\alpha_{6}: \mathbb{R}_{+} \rightarrow \mathbb{R}_{+}$by

$$
\alpha_{6}(s):=\frac{2}{s} \int_{s / 2}^{s} \alpha_{5}(t) d t \quad \forall s>0, \quad \alpha_{6}(0):=\lim _{s \downarrow 0} \alpha_{6}(s)=0 .
$$

Since $\alpha_{5} \in \mathcal{K}_{\infty}$, we have $\alpha_{5}(s / 2) \leq \alpha_{6}(s) \leq \alpha_{5}(s)$ for all $s \in \mathbb{R}_{+}$and, moreover, $\alpha_{6}$ is differentiable on $(0, \infty)$ with derivative $\alpha_{6}^{\prime}(s) \geq 0$ for all $s \in(0, \infty)$. Now 
define $\alpha_{7}: \mathbb{R}_{+} \rightarrow \mathbb{R}_{+}$by $\alpha_{7}(s):=\min \{1, s\} \alpha_{6}(s)$. Clearly, $\alpha_{7}$ is locally Lipschitz, $\alpha_{7}(0)=0$ and $0<\alpha_{7}(s) \leq \alpha_{5}(s)$ for all $s>0$. Define the locally Lipschitz function

$$
Z: \mathbb{R} \rightarrow \mathbb{R}, \quad \zeta \mapsto Z(\zeta):= \begin{cases}-\alpha_{7}(\zeta) / 2, & \zeta \geq 0 \\ \alpha_{7}(-\zeta) / 2, & \zeta<0\end{cases}
$$

and consider the scalar system

$$
\dot{z}(t)=Z(z(t)) .
$$

Since $Z(0)=0$ and $\zeta Z(\zeta)=-|\zeta| \alpha_{7}(|\zeta|) / 2<0$ for all $\zeta \neq 0$, it follows that 0 is a globally asymptotically stable equilibrium of this system which, together with the local Lipschitz property of $Z$, ensures the existence of a continuous global semiflow $\beta: \mathbb{R}_{+} \times \mathbb{R} \rightarrow \mathbb{R}$ (and so, for each $z^{0} \in \mathbb{R}, z: \mathbb{R}_{+} \rightarrow \mathbb{R}, t \mapsto \beta\left(t, z^{0}\right)$, is the unique global solution of the initial-value problem $\dot{z}=Z(z), z(0)=z^{0}$; moreover, $\beta\left(t, z^{0}\right) \rightarrow 0$ as $\left.t \rightarrow \infty\right)$. Let $\beta_{0}:=\left.\beta\right|_{\mathbb{R}_{+} \times \mathbb{R}_{+}}$be the restriction of $\beta$ to $\mathbb{R}_{+} \times \mathbb{R}_{+}$. Evidently, $\beta_{0} \in \mathcal{K} \mathcal{L}$. Now define $\beta_{1} \in \mathcal{K} \mathcal{L}$ by

$$
\beta_{1}(t, s):=\alpha_{1}^{-1}\left(\beta_{0}\left(t, \alpha_{2}(s)\right)\right),
$$

and define $\beta_{2} \in \mathcal{K}_{\infty}$ by

$$
\left.\beta_{2}(s):=\left(\alpha_{1}^{-1} \circ \alpha_{2} \circ \alpha_{3}^{-1}\right)\left(2 \alpha_{4}(s)\right)\right) .
$$

Let $x^{0}$ and $\Delta \in \mathcal{B}$ be arbitrary, and let $x$ be a global solution of (2.1). Let $t \in \mathbb{R}_{+}$ be arbitrary. By (5.30), we have

$$
\begin{array}{r}
(V \circ x)^{\prime}(\tau) \leq-\alpha_{3}(\|x(\tau)\|)+\alpha_{4}(|\Delta(\tau)|) \leq-\alpha_{3}(\|x(\tau)\|)+\alpha_{4}\left(\|\Delta\|_{L^{\infty}[0, t]}\right) \\
\text { for a.e. } \tau \in[0, t] .
\end{array}
$$

Clearly,

$$
\left.V(x(t)) \leq\left(\alpha_{2} \circ \alpha_{3}^{-1}\right)\right)\left(2 \alpha_{4}\left(\|\Delta\|_{L^{\infty}[0, t]}\right) \quad \Longrightarrow \quad\|x(t)\| \leq \beta_{2}\left(\|\Delta\|_{L^{\infty}[0, t]}\right) .\right.
$$

Moreover,

$$
V(x(t))>\left(\alpha_{2} \circ \alpha_{3}^{-1}\right)\left(2 \alpha_{4}\left(\|\Delta\|_{L^{\infty}[0, t]}\right)\right) \quad \Longrightarrow \quad \alpha_{3}(\|x(t)\|)>2 \alpha_{4}\left(\|\Delta\|_{L^{\infty}[0, t]}\right),
$$

which, together with (5.32), yields

$$
\begin{aligned}
& V(x(t))>\left(\alpha_{2} \circ \alpha_{3}^{-1}\right)\left(2 \alpha_{4}\left(\|\Delta\|_{L^{\infty}[0, t]}\right)\right) \\
& \Longrightarrow(V \circ x)^{\prime}(\tau)<-\frac{1}{2} \alpha_{3}(\|x(\tau)\|) \leq-\frac{1}{2} \alpha_{5}(V(x(\tau))) \leq-\frac{1}{2} \alpha_{7}(V(x(\tau))) \\
&=Z(V(x(\tau))) \text { for a.e. } \tau \in[0, t]
\end{aligned}
$$

and so

$$
\begin{aligned}
V(x(t))>\left(\alpha_{2} \circ \alpha_{3}^{-1}\right) & \left(2 \alpha_{4}\left(\|\Delta\|_{L^{\infty}[0, t]}\right)\right. \\
& \Longrightarrow V(x(t)) \leq \beta_{0}\left(t, V\left(x^{0}\right)\right) \Longrightarrow\|x(t)\| \leq \beta_{1}\left(t,\left\|x^{0}\right\|\right) .
\end{aligned}
$$

Therefore, $\|x(t)\| \leq \max \left\{\beta_{1}\left(t,\left\|x^{0}\right\|\right), \beta_{2}\left(\|\Delta\|_{L^{\infty}[0, t]}\right)\right\}$ for all $t \in \mathbb{R}_{+}$.

6. Hysteretic feedback systems. We return to the feedback interconnection of Figure 1.2, but now in a single-input $(t \mapsto d(t) \in \mathbb{R})$, single-output $(t \mapsto y(t) \in \mathbb{R})$ setting and with a hysteresis operator $F$ in the feedback path, as shown in Figure 6.1. We deem an operator $F: C\left(\mathbb{R}_{+}\right) \rightarrow C\left(\mathbb{R}_{+}\right)$is a hysteresis operator if it is both causal and rate independent. By rate independence we mean that $F(y \circ \zeta)=(F y) \circ \zeta$ 


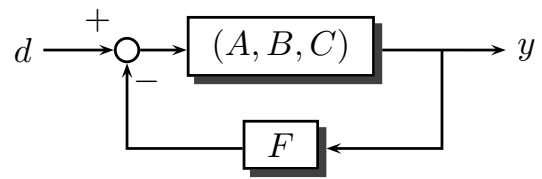

FIG. 6.1. Interconnection of a linear system $(A, B, C)$ and a hysteresis operator $F$

for every $y \in C\left(\mathbb{R}_{+}\right)$and every time transformation $\zeta: \mathbb{R}_{+} \rightarrow \mathbb{R}_{+}$(that is, a continuous, non-decreasing and surjective map). Conditions on $F$ which ensure wellposedness of the feedback interconnection (existence and uniqueness of solutions of the associated initial-value problem) are expounded in, for example, [16] and [17]. Whilst, in principle, the ensuing analysis is applicable in the context of any causal operator $F$ that can be embedded in a set-valued map $\Phi \in \mathcal{U}$, for clarity of presentation we focus on the class of Preisach operators.

Preisach and Prandtl hysteresis. The Preisach operator described in this section encompasses both backlash and Prandtl operators. It can model complex hysteresis effects: for example, nested loops in input-output characteristics. A basic building block for these operators is the backlash operator. A discussion of the backlash operator (also called play operator) can be found in a number of references, see for example [5], [14] and [15]. Let $\sigma \in \mathbb{R}_{+}$and introduce the function $b_{\sigma}: \mathbb{R}^{2} \rightarrow \mathbb{R}$ given by

$$
b_{\sigma}\left(v_{1}, v_{2}\right):=\max \left\{v_{1}-\sigma, \min \left\{v_{1}+\sigma, v_{2}\right\}\right\}= \begin{cases}v_{1}-\sigma, & \text { if } v_{2}<v_{1}-\sigma \\ v_{2}, & \text { if } v_{2} \in\left[v_{1}-\sigma, v_{1}+\sigma\right] \\ v_{1}+\sigma, & \text { if } v_{2}>v_{1}+\sigma\end{cases}
$$

Let $C_{\mathrm{pm}}\left(\mathbb{R}_{+}\right)$denote the space of continuous piecewise monotone functions defined on $\mathbb{R}_{+}$. For all $\sigma \in \mathbb{R}_{+}$and $\zeta \in \mathbb{R}$, define the operator $\mathcal{B}_{\sigma, \zeta}: C_{\mathrm{pm}}\left(\mathbb{R}_{+}\right) \rightarrow C\left(\mathbb{R}_{+}\right)$ by

$$
\mathcal{B}_{\sigma, \zeta}(y)(t)= \begin{cases}b_{\sigma}(y(0), \zeta) & \text { for } t=0 \\ b_{\sigma}\left(y(t),\left(\mathcal{B}_{\sigma, \zeta}(u)\right)\left(t_{i}\right)\right) & \text { for } t_{i}<t \leq t_{i+1}, i=0,1,2, \ldots\end{cases}
$$

where $0=t_{0}<t_{1}<t_{2}<\ldots, \lim _{n \rightarrow \infty} t_{n}=\infty$ and $u$ is monotone on each interval $\left[t_{i}, t_{i+1}\right]$. We remark that $\zeta$ plays the role of an "initial state". It is not difficult to show that the definition is independent of the choice of the partition $\left(t_{i}\right)$. Figure 6.2 illustrates how $\mathcal{B}_{\sigma, \zeta}$ acts. It is well-known that $\mathcal{B}_{\sigma, \zeta}$ extends to a Lipschitz

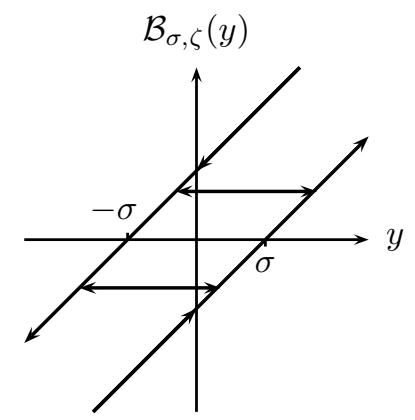

FIG. 6.2. Backlash hysteresis

continuous hysteresis operator on $C\left(\mathbb{R}_{+}\right)$(with Lipschitz constant $L=1$ ), the socalled backlash operator, which we shall denote by the same symbol $\mathcal{B}_{\sigma, \zeta}$.

Let $\xi: \mathbb{R}_{+} \rightarrow \mathbb{R}$ be a compactly supported and globally Lipschitz function with Lipschitz constant 1 . Let $\mu$ be a regular signed Borel measure on $\mathbb{R}_{+}$. Denoting 
Lebesgue measure on $\mathbb{R}$ by $\mu_{L}$, let $w: \mathbb{R} \times \mathbb{R}_{+} \rightarrow \mathbb{R}$ be a locally $\left(\mu_{L} \otimes \mu\right)$-integrable function and let $w_{0} \in \mathbb{R}$. The operator $\mathcal{P}_{\xi}: C\left(\mathbb{R}_{+}\right) \rightarrow C\left(\mathbb{R}_{+}\right)$defined by

$$
\begin{aligned}
&\left(\mathcal{P}_{\xi}(y)\right)(t)=\int_{0}^{\infty} \int_{0}^{\left(\mathcal{B}_{\sigma, \xi(\sigma)}(y)\right)(t)} w(s, \sigma) \mu_{L}(\mathrm{~d} s) \mu(\mathrm{d} \sigma)+w_{0} \\
& \forall u \in C\left(\mathbb{R}_{+}\right), \quad \forall t \in \mathbb{R}_{+},
\end{aligned}
$$

is called a Preisach operator: this definition is equivalent to that adopted in [5, Section 2.4]. It is well-known that $\mathcal{P}_{\xi}$ is a hysteresis operator (this follows from the fact that $\mathcal{B}_{\sigma, \xi(\sigma)}$ is a hysteresis operator for every $\left.\sigma \geq 0\right)$. Under the assumption that the measure $\mu$ is finite and $w$ is essentially bounded, the operator $\mathcal{P}_{\xi}$ is Lipschitz continuous with Lipschitz constant $L=|\mu|\left(\mathbb{R}_{+}\right)\|w\|_{\infty}$ (see [15]) in the sense that

$$
\sup _{t \in \mathbb{R}_{+}}\left|\mathcal{P}_{\xi}\left(y_{1}\right)(t)-\mathcal{P}_{\xi}\left(y_{2}\right)(t)\right| \leq L \sup _{t \in \mathbb{R}_{+}}\left|y_{1}(t)-y_{2}(t)\right| \quad \forall y_{1}, y_{2} \in C\left(\mathbb{R}_{+}\right) .
$$

This property ensures the well-posedness of the feedback interconnection.

Setting $w(\cdot, \cdot)=1$ and $w_{0}=0$ in (6.1), we obtain the Prandtl operator $\mathcal{P}_{\xi}$ : $C\left(\mathbb{R}_{+}\right) \rightarrow C\left(\mathbb{R}_{+}\right)$defined by

$$
\mathcal{P}_{\xi}(y)(t)=\int_{0}^{\infty}\left(\mathcal{B}_{\sigma, \xi(\sigma)}(y)\right)(t) \mu(\mathrm{d} \sigma) \quad \forall u \in C\left(\mathbb{R}_{+}\right), \quad \forall t \in \mathbb{R}_{+} .
$$

For $\xi \equiv 0$ and $\mu$ given by $\mu(E)=\int_{E} \chi_{[0,5]}(\sigma) \mathrm{d} \sigma$ (where $\chi_{[0,5]}$ denotes the indicator function of the interval $[0,5])$, the Prandtl operator is illustrated in Figure 6.3.
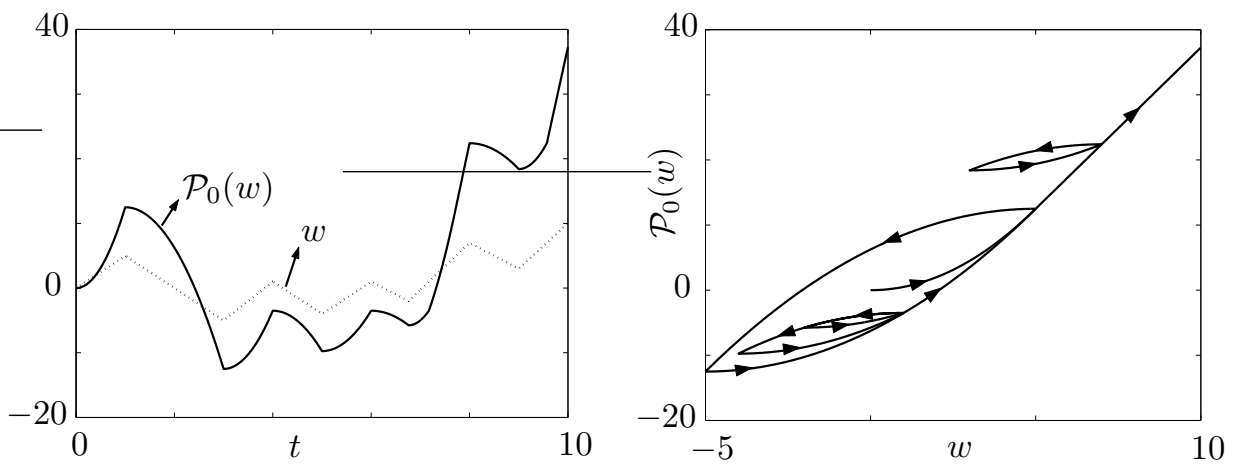

FIG. 6.3. Example of Prandtl hysteresis

The next proposition identifies conditions under which the Preisach operator (6.1) satisfies a generalized sector bound. For simplicity, we assume that the measure $\mu$ and the function $w$ are non-negative (an important case in applications), although the proposition can be extended to signed measures $\mu$ and sign-indefinite functions $w$.

Proposition 6.1. Let $\mathcal{P}_{\xi}$ be the Preisach operator defined in (6.1). Assume that the measure $\mu$ is non-negative, $a_{1}:=\mu\left(\mathbb{R}_{+}\right)<\infty, a_{2}:=\int_{0}^{\infty} \sigma \mu(\mathrm{d} \sigma)<\infty$, $b_{1}:=\operatorname{essinf}_{(s, \sigma) \in \mathbb{R} \times \mathbb{R}_{+}} w(s, \sigma) \geq 0, b_{2}:=\operatorname{ess}_{\sup _{(s, \sigma) \in \mathbb{R} \times \mathbb{R}_{+}} w(s, \sigma)<\infty \text { and set }}$

$$
a_{\mathcal{P}}:=a_{1} b_{1}, \quad b_{\mathcal{P}}:=a_{1} b_{2}, \quad c_{\mathcal{P}}:=a_{2} b_{2}+\left|w_{0}\right| .
$$

Then, for all $y \in C\left(\mathbb{R}_{+}\right)$and all $t \in \mathbb{R}_{+}$

$$
\begin{aligned}
& y(t) \geq 0 \Longrightarrow a_{\mathcal{P}} y(t)-c_{\mathcal{P}} \leq\left(\mathcal{P}_{\xi}(y)\right)(t) \leq b_{\mathcal{P}} y(t)+c_{\mathcal{P}}, \\
& y(t) \leq 0 \Longrightarrow b_{\mathcal{P}} y(t)-c_{\mathcal{P}} \leq\left(\mathcal{P}_{\xi}(y)\right)(t) \leq a_{\mathcal{P}} y(t)+c_{\mathcal{P}},
\end{aligned}
$$


and, furthermore, for every $\eta>0$,

$$
|y(t)| \geq c_{\mathcal{P}} / \eta \Longrightarrow\left(a_{\mathcal{P}}-\eta\right) y^{2}(t) \leq\left(\mathcal{P}_{\xi}(y)\right)(t) y(t) \leq\left(b_{\mathcal{P}}+\eta\right) y^{2}(t) .
$$

Proof. Let $y \in C\left(\mathbb{R}_{+}\right)$and $t \in \mathbb{R}_{+}$be arbitrary. Note initially that, by the definition of the backlash operator,

$$
\left(\mathcal{B}_{\sigma, \xi(\sigma)}(y)\right)(t) \in[y(t)-\sigma, y(t)+\sigma] \forall \sigma \in \mathbb{R}_{+} .
$$

Case 1. Assume $y(t) \geq 0$. Writing $E_{1}:=[0, y(t)]$ and $E_{2}:=(y(t), \infty)$, we have

$$
\begin{aligned}
\left(\mathcal{P}_{\xi} y\right)(t) & \geq\left(\int_{E_{1}}+\int_{E_{2}}\right) \int_{0}^{y(t)-\sigma} w(s, \sigma) \mu_{L}(\mathrm{~d} s) \mu(\mathrm{d} \sigma)-\left|w_{0}\right| \\
& \geq b_{1} \int_{E_{1}}(y(t)-\sigma) \mu(\mathrm{d} \sigma)+b_{2} \int_{E_{2}}(y(t)-\sigma) \mu(\mathrm{d} \sigma)-\left|w_{0}\right| \\
& =\left(b_{1} \mu\left(E_{1}\right)+b_{2} \mu\left(E_{2}\right)\right) y(t)-b_{1} \int_{E_{1}} \sigma \mu(\mathrm{d} \sigma)-b_{2} \int_{E_{2}} \sigma \mu(\mathrm{d} \sigma)-\left|w_{0}\right| \\
& \geq a_{1} b_{1} y(t)-a_{2} b_{2}-\left|w_{0}\right|=a_{\mathcal{P}} y(t)-c_{\mathcal{P}} .
\end{aligned}
$$

Moreover,

$$
\begin{aligned}
\left(\mathcal{P}_{\xi} y\right)(t) & \leq \int_{0}^{\infty} \int_{0}^{y(t)+\sigma} w(s, \sigma) \mu_{L}(\mathrm{~d} s) \mu(\mathrm{d} \sigma)+\left|w_{0}\right| \\
& \leq b_{2} \int_{0}^{\infty}(y(t)+\sigma) \mu(\mathrm{d} \sigma)+\left|w_{0}\right| \leq a_{1} b_{2} y(t)+a_{2} b_{2}+\left|w^{0}\right|=b_{\mathcal{P}} y(t)+c_{\mathcal{P}} .
\end{aligned}
$$

This establishes (6.4).

Case 2. Now assume $y(t) \leq 0$. The argument used in Case 1 applies mutatis mutandis to conclude (6.5).

Finally, the inequality (6.6) is a straightforward consequence of (6.4) and (6.5). $\mathrm{C}$ For example, the Prandtl operator in Figure 6.3 satisfies the hypotheses of Proposition 6.1.

Let $\mathcal{P}_{\xi}$ be a Preisach operator satisfying the hypotheses of Proposition 6.1. Let $a_{\mathcal{P}}$, $b_{\mathcal{P}}$ and $c_{\mathcal{P}}$ be given by (6.3) and define $\Phi, \tilde{\Phi} \in \mathcal{U}$ by

$$
\begin{gathered}
\Phi(y):= \begin{cases}\left\{v \in \mathbb{R} \mid a_{\mathcal{P}} y-c_{\mathcal{P}} \leq v \leq b_{\mathcal{P}} y+c_{\mathcal{P}}\right\}, & y \geq 0 \\
\left\{v \in \mathbb{R} \mid b_{\mathcal{P}} y-c_{\mathcal{P}} \leq v \leq a_{\mathcal{P}} y+c_{\mathcal{P}}\right\}, & y<0 .\end{cases} \\
\tilde{\Phi}(y):=\left\{v \in \mathbb{R} \mid\left(a_{\mathcal{P}}-\eta\right) y^{2} \leq v y \leq\left(b_{\mathcal{P}}+\eta\right) y^{2}\right\},
\end{gathered}
$$

where $\eta>0$. In view of (6.4) and (6.5),

$$
y \in C\left(\mathbb{R}_{+}\right) \quad \Longrightarrow \quad\left(\mathcal{P}_{\xi}(y)\right)(t) \in \Phi(y(t)) \forall t \in \mathbb{R}_{+} .
$$

Moreover, writing $K:=\left[-c_{\mathcal{P}} / \eta, c_{\mathcal{P}} / \eta\right]$, we have

$$
\Phi(y) \subset \tilde{\Phi}(y) \quad \forall y \in \mathbb{R} \backslash K \text { and } E:=\sup _{y \in K} \sup _{v \in \Phi(y)} \inf _{\tilde{v} \in \tilde{\Phi}(y)}|v-\tilde{v}|=c_{\mathcal{P}} .
$$

Let the linear system $(A, B, C)$ (with transfer function $G$ ) be stabilizable and detectable. Write $a:=a_{\mathcal{P}}-\eta, b:=b_{\mathcal{P}}+\eta$ and assume that $G /(1+a G) \in H^{\infty}$ and, for some $\delta \in(0,1),(1+b G) /(1+a G)-\delta$ is positive real. Then hypothesis (H1) holds with $m=1$ and $\tilde{\Phi}$ replacing $\Phi$. 
Example. As a concrete example, consider a mechanical system with damping coefficient $\gamma>0$ and hysteretic restoring force in the form of backlash, with real parameters $\sigma>0$ and $\zeta$ :

$$
\ddot{y}(t)+\gamma \dot{y}(t)+\mathcal{B}_{\sigma, \zeta}(y)(t)=d(t) .
$$

Setting $w(\cdot, \cdot):=1, w^{0}=0, \mu:=\delta_{\sigma}$ (the Dirac measure with support $\{\sigma\}$ ) and $\xi(\cdot):=\zeta$ in (6.1), we see that $\mathcal{B}_{\sigma, \zeta}=\mathcal{P}_{\xi}$. In this case, and in the notation of Proposition 6.1, we have $a_{1}=b_{1}=b_{2}=a_{\mathcal{P}}=b_{\mathcal{P}}=1$ and $a_{2}=c_{\mathcal{P}}=\sigma$. Choosing $\eta \in(0,1)$, we have $0<a<b$, where, as before, $a=a_{\mathcal{P}}-\eta$ and $b=b_{\mathcal{P}}+\eta$ and, by Proposition 6.1,

$$
|y(t)| \geq \sigma / \eta \Longrightarrow a y^{2}(t) \leq\left(\mathcal{B}_{\sigma, \xi}(y)\right)(t) y(t) \leq b y^{2}(t) .
$$

The transfer function $G$ is given by $G(s)=1 /\left(s^{2}+\gamma s\right), G /(1+a G)$ is given by $1 /\left(s^{2}+\gamma s+a\right)$ and $(1+b G) /(1+a G)-\delta$ is given by $(1-\delta)+2 \eta /\left(s^{2}+\gamma s+a\right)$ : clearly $G /(1+a G) \in H^{\infty}$ and a straightforward calculation reveals that, for all $\eta>0$ sufficiently small, $(1+b G) /(1+a G)-\delta$ is positive real.

Returning to the general setting, we are now in a position to invoke Corollary 3.6 to conclude properties of solutions of the single-input, single-output, functional differential equation

$$
\dot{x}(t)=A x(t)+B\left[d(t)-\left(\mathcal{P}_{\xi}(C x)\right)(t)\right], \quad x(0)=x^{0} .
$$

We reiterate that, for each $x^{0} \in \mathbb{R}^{n}$ and $d \in L_{\text {loc }}^{\infty}\left(\mathbb{R}_{+}\right)$, (6.8) has unique global solution. An application of Corollary 3.6 (with $\Delta(t)=\{d(t)\}$ for all $t \in \mathbb{R}_{+}$) yields the existence of constants $\varepsilon, c_{1}, c_{2}>0$ such that, for every global solution $x$,

$$
\|x(t)\| \leq c_{1} e^{-\varepsilon t}\left\|x^{0}\right\|+c_{2}\left(\|d\|_{L^{\infty}[0, t]}+c_{\mathcal{P}}\right) \quad \forall t \in \mathbb{R}_{+},
$$

showing in particular that $(6.8)$ is input-to-state stable with bias $c_{\mathcal{P}}$. Furthermore, by Lemma 3.3 ,

$$
\lim _{t \rightarrow \infty} d(t)=0 \Longrightarrow \limsup _{t \rightarrow \infty}\|x(t)\| \leq c_{2} c_{\mathcal{P}} .
$$

We emphasize that the convergence $d(t) \rightarrow 0$ as $t \rightarrow \infty$ does in general not imply convergence of $x(t)$ as $t \rightarrow \infty$. To see this, consider again the mechanical example (6.7). Then, for every $\gamma>0$, there exist constants $\varepsilon, c_{1}, c_{2}>0$ such that (6.9) and (6.10) hold (with $x(t)=(y(t), \dot{y}(t))$ and $\left.c_{\mathcal{P}}=\sigma\right)$. However, we know from [17, Example 4.8] that, if $d=0$ and $\gamma \in(1,2)$, then for all initial conditions, $\lim \sup _{t \rightarrow \infty} y(t)=\sigma$ and $\liminf _{t \rightarrow \infty} y(t)=-\sigma$ (equivalently, $y$ has $\omega$-limit set $[-\sigma, \sigma])$, showing in particular that $x(t)=(y(t), \dot{y}(t))$ does not converge as $t \rightarrow \infty$.

7. Quantized feedback systems. Let $(A, B, C)$ be a minimal realization of a linear, single-input, single-output system with transfer function $G$. Let $f: \mathbb{R} \rightarrow \mathbb{R}$ be a continuous static nonlinearity with the following property.

(Q1) There exist $\varphi \in \mathcal{K}_{\infty}$ and a number $b>0$ such that

$$
\varphi(|y|)|y| \leq f(y) y \leq b y^{2} \quad \forall y \in \mathbb{R} .
$$

Furthermore, we impose the following assumption.

(Q2) There exists $\kappa \in[0,1 / b)$ such that $\kappa+G$ is positive real.

From (Q1) and (Q2), it follows that (H3) holds with $\Phi(y)=\{f(y)\}$ and $\delta=\kappa b \in$ $[0,1)$. Consequently, by Theorem 3.5 , the system

$$
\dot{x}(t)=A x(t)+B(d(t)-f(C x(t))), \quad x(0)=x^{0} \in \mathbb{R}^{n}, \quad d \in L_{\mathrm{loc}}^{\infty}\left(\mathbb{R}_{+}\right),
$$


is input-to-state stable. Now consider (7.1) subject to quantization of the output $y=C x$, that is, the system

$$
\dot{x}(t)=A x(t)+B\left(d(t)-\left(f \circ q_{\gamma}\right)(C x(t))\right), \quad x(0)=x^{0} \in \mathbb{R}^{n}, \quad d \in L_{\mathrm{loc}}^{\infty}\left(\mathbb{R}_{+}\right),
$$

where $q_{\gamma}: \mathbb{R} \rightarrow \mathbb{R}$, parameterized by $\gamma>0$, is a uniform quantizer (see Figure 7.1 ) given by

$$
q_{\gamma}(y)=2(m+1) \gamma \quad \forall y \in((2 m+1) \gamma,(2 m+3) \gamma] \quad \forall m \in \mathbb{Z} .
$$

We interpret the differential equation (with discontinuous righthand side) in (7.1)

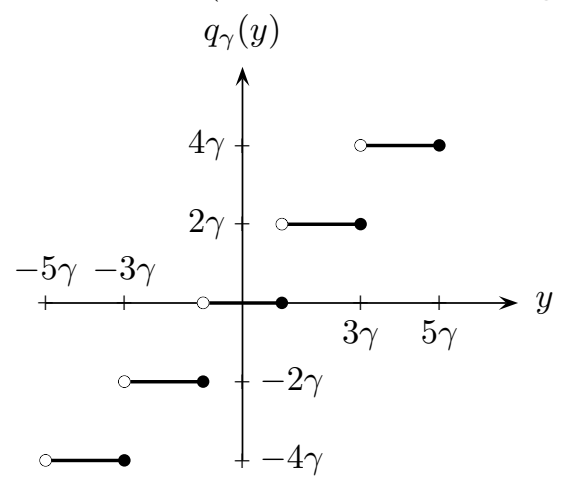

FIG. 7.1. Uniform quantizer

in a set-valued sense by embedding the quantizer $q_{\gamma}$ in the set-valued map $Q_{\gamma} \in \mathcal{U}$ defined by

$$
Q_{\gamma}(y):= \begin{cases}\left\{q_{\gamma}(y)\right\}, & y \in((2 m+1) \gamma,(2 m+3) \gamma), \quad m \in \mathbb{Z} \\ {[2 m \gamma, 2(m+1) \gamma],} & y=(2 m+1) \gamma, \quad m \in \mathbb{Z}\end{cases}
$$

and subsuming (7.2) in the differential inclusion

$$
\dot{x}(t)-A x(t) \in B\left(\Delta(t)-\Phi_{\gamma}(C x(t))\right), \quad x(0)=x^{0} \in \mathbb{R}^{n}, \quad \Delta \in \mathcal{B},
$$

where $\Delta: t \mapsto\{d(t)\}$ and $\Phi_{\gamma} \in \mathcal{U}$ is given by

$$
\Phi_{\gamma}(y):=f\left(Q_{\gamma}(y)\right)=\left\{f(\xi) \mid \xi \in Q_{\gamma}(y)\right\}
$$

Choose $\varepsilon \in(0,1)$ sufficiently small so that $(1+\varepsilon) \kappa<1 / b$. Write $\tilde{b}:=(1+\varepsilon) b$ and define $\tilde{\varphi} \in \mathcal{K}_{\infty}$ by $\tilde{\varphi}(s):=\varphi((1-\varepsilon) s)$ for all $s \in \mathbb{R}_{+}$.

Lemma 7.1. There exists $M \in \mathbb{N}$ such that, for every $\gamma>0$,

$$
y \in \mathbb{R},|y| \geq \gamma M, v \in \Phi_{\gamma}(y) \quad \Longrightarrow \quad \tilde{\varphi}(|y|)|y| \leq y v \leq \tilde{b} y^{2}
$$

Proof. Observe that, for all $m \in \mathbb{N}$,

$$
\frac{2 m+2}{2 m+3} \leq \frac{w}{y} \leq \frac{2 m+4}{2 m+1} \quad \forall w \in Q_{\gamma}(y) \quad \forall y \in((2 m+1) \gamma,(2 m+3) \gamma] .
$$

Therefore, there exists $M \in \mathbb{N}$ such that

$$
(1-\varepsilon) y^{2} \leq w y \leq(1+\varepsilon) y^{2} \quad \forall w \in Q_{\gamma}(y) \quad \forall y \geq \gamma M
$$

Since $Q_{\gamma}$ has odd symmetry $\left(Q_{\gamma}(y)=-Q_{\gamma}(-y)\right)$, it immediately follows that

$$
(1-\varepsilon) y^{2} \leq w y \leq(1+\varepsilon) y^{2} \quad \forall w \in Q_{\gamma}(y) \quad \forall|y| \geq \gamma M
$$


Let $y$ be such that $|y| \geq \gamma M$ and let $v \in \Phi_{\gamma}(y)$. Then $v=f(w)$ for some $w \in Q_{\gamma}(y)$. Invoking (Q1) and (7.4), it follows that

$$
\varphi(|w|)|y|=\varphi(|w|)|w| \frac{y}{w} \leq f(w) w \frac{y}{w}=f(w) y=v y \leq b w y \leq(1+\varepsilon) b y^{2}=\tilde{b} y^{2} .
$$

Since $\varphi(|w|)=\varphi(|w||y| /|y|)=\varphi(w y /|y|)$ and invoking (7.4) and (7.5), we have

$$
\tilde{\varphi}(|y|)|y|=\varphi((1-\varepsilon)|y|)|y| \leq \varphi(|w|)|y| \leq v y \leq \tilde{b} y^{2},
$$

This completes the proof.

Let $M \in \mathbb{N}$ be as in Lemma 7.1 and define $\tilde{\Phi} \in \mathcal{U}$ by

$$
\tilde{\Phi}(y):=\left\{\begin{array}{cc}
{[\tilde{\varphi}(|y|), \tilde{b}|y|],} & |y| \geq 0 \\
{[-\tilde{b}|y|,-\tilde{\varphi}(|y|)],} & y<0 .
\end{array}\right.
$$

Clearly,

$$
y \in \mathbb{R}, v \in \tilde{\Phi}(y) \quad \Longrightarrow \quad \max \left\{\tilde{\varphi}(|y|)|y|, v^{2} / \tilde{b}\right\} \leq y v,
$$

and, by Lemma 7.1, we also have $\Phi_{\gamma}(y) \subset \tilde{\Phi}(y)$ for all $y \in \mathbb{R} \backslash[-\gamma M, \gamma M]$. Moreover, by (Q2) (and recalling that $\kappa<\tilde{b})),(\delta / \tilde{b})+G$ is positive real for every $\delta \in[\kappa \tilde{b}, 1)$. We are now in a position to invoke Corollary 3.7 (with $K=[-\gamma M, \gamma M]$ ) to conclude the existence of $\beta_{1} \in \mathcal{K} \mathcal{L}$ and $\beta_{2} \in \mathcal{K}_{\infty}$, which do not depend on $\gamma>0$ (recall Remark 3.8), such that, for all $\gamma>0$, all $x^{0} \in \mathbb{R}^{n}$ and all $d \in L_{\text {loc }}^{\infty}\left(\mathbb{R}_{+}\right)$, every global solution of (7.3), with $\Delta: t \mapsto\{d(t)\}$ satisfies

$$
\|x(t)\| \leq \max \left\{\beta_{1}\left(t,\left\|x^{0}\right\|\right), \beta_{2}\left(\|d\|_{L^{\infty}[0, t]}+E_{\gamma}\right)\right\} \quad \forall t \in \mathbb{R}_{+},
$$

where $E_{\gamma}:=\sup _{|y| \leq \gamma M} \sup _{v \in \Phi_{\gamma}(y)} \inf _{\tilde{v} \in \tilde{\Phi}_{\gamma}(y)}|v-\tilde{v}|$. Noting that $E_{\gamma} \rightarrow 0$ as $\gamma \downarrow 0$ (if $f$ is locally Lipschitz, then $E_{\gamma}=O(\gamma)$ as $\gamma \downarrow 0$ ), we may conclude robustness with respect to quantization in the sense that the quantized feedback system is such that the unbiased ISS property of the unquantized system (7.1) is approached as $\gamma \downarrow 0$.

8. Conclusion. Feedback interconnections consisting of a linear system in the forward path and a nonlinearity in the feedback path have been considered. Adopting a differential inclusions framework, nonlinearities of considerable generality are encompassed, including inter alia both hysteresis operators and quantization operators. Conditions on the linear and nonlinear components have been identified (in Theorems 3.4 and 3.5) under which input-to-state stability (and a fortiori global asymptotic stability of the zero state) of the feedback interconnection is assured. The results of this paper are in the spirit of absolute stability theory: in particular, when specialized appropriately, classical absolute stability results pertaining to the circle criterion are recovered. In Corollaries 3.6 and 3.7, hypotheses are imposed on the nonlinearities (namely, generalized sector conditions), considerably weaker than those posited in Theorems 3.4 and 3.5, under which input-to-state stability with bias (and a fortiori asymptotic stability of a compact neighbourhood of the zero state) may be concluded. Applications of the results to systems with hysteresis and to systems with output quantization have been detailed.

\section{REFERENCES}

[1] M. Arcak \& A. Teel, Input-to-state stability for a class of Lurie systems, Automatica, 38, 1945-1949, 2002.

[2] J.P. Aubin \& A. Cellina, Differential Inclusions, Springer Verlag, Berlin, 1984.

[3] N.A. Barabanov \& V.A.Yakubovich. Absolute stability of control systems with one hysteresis nonlinearity. Automation and Remote Control, 12, 5-12, 1979. 
[4] R.W. Brockett \& D. Liberzon. Quantized feedback stabilization of linear systems, IEEE Trans. Automatic Control, 45, 1279-1289, 2000.

[5] M. Brokate \& J. Sprekels, Hysteresis and Phase Transitions, Springer Verlag, New York, 1996.

[6] K. Deimling, Multivalued Differential Equations, de Gruyter, Berlin, 1992.

[7] C.A. Desoer \& M. Vidyasagar, Feedback Systems: Input-Output Properties, Academic Press, New York, 1975.

[8] M. Fu \& L. Xie, The sector bound approach to quantized feedback control, IEEE Trans. Automatic Control, 50, 1698-1711, 2005.

[9] R.B. Gorbet, K.A. Morris \& D.W.L. Wang. Passivity-based stability and control of hysteresis in smart actuators, IEEE Trans. Control Systems Technology, 9, 5-16, 2001.

[10] W. Hahn, Stability of Motion, Springer-Verlag, Berlin, 1967.

[11] B. Jayawardhana, H. Logemann and E.P. Ryan, Infinite-dimensional feedback systems: the circle criterion and input-to-state stability, submitted for publication (2008) (www.maths.bath.ac.uk/ hl/PUBLICATIONS/jayawardhana_ryan_08.pdf)

[12] U. Jönson. Stability of uncertain systems with hysteresis nonlinearities. International Journal of Robust and Nonlinear Control, 8,279-293, 1998.

[13] H.K. Khalil. Nonlinear Systems, 3rd edition, Prentice-Hall, Upper Saddle River, NJ, 2002.

[14] M.A. Krasnosel'skii \& A.V. Pokrovskii, Systems with Hysteresis. Springer Verlag, Berlin, 1989.

[15] H. Logemann \& A.D. Mawby, Low-gain integral control of infinite-dimensional regular linear systems subject to input hysteresis, in F. Colonius et al. (eds.) Advances in Mathematical Systems Theory, pp. 255-293, Birkhäuser, Boston, 2001.

[16] H. Logemann \& E.P. Ryan, Systems with hysteresis in the feedback loop: existence, regularity and asymptotic behaviour of solutions, ESAIM Control, Optimiz. \& Calculus of Variations, 9, 169-196, 2003.

[17] H. Logemann, E.P. Ryan \& I. Shvartsman, A class of differential-delay systems with hysteresis: asymptotic behaviour of solutions, Nonlinear Analysis, 69, 363-391, 2008.

[18] A.I. Lur'e \& V.N. Postnikov, On the theory of stability of control systems, Applied Math. $\&$ Mechanics, 8, 246-248, 1944 (in Russian).

[19] K.S. Narendra \& J.H. Taylor, Frequency Criteria for Absolute Stability, Academic Press, New York, 1973.

[20] E.D. Sontag, Input to state stability: basic concepts and results, in P. Nistri and G. Stefani (eds.) Nonlinear and Optimal Control Theory, pp. 163-220, Springer Verlag, Berlin, 2006.

[21] M. Vidyasagar, Nonlinear Systems Analysis, 2nd edition, Prentice-Hall, Englewood Cliffs, NJ, 1993.

[22] R. Vinter. Optimal Control, Birkhäuser, Boston, 2000.

[23] V.A. Yakubovich, G.A. Leonov \& A.Kh. Gelig. Stability of Stationary Sets in Control Systems with Discontinuous Nonlinearities, World Scientific, Singapore, 2004. 